\title{
Impact of the electronic environment in carbazole-appended $o$-carboranyl compounds on the intramolecular-charge-transfer-based radiative decay efficiency
}

\author{
Seok Ho Lee, ${ }^{\dagger}$ Min Sik Mun, ${ }^{\dagger}$ Ji Hye Lee, Sehee Im, Wonchul Lee, Hyonseok Hwang, \\ and Kang Mun Lee*
}

Department of Chemistry, Institute for Molecular Science and Fusion Technology, Kangwon National University, Chuncheon, Gangwon 24341, Republic of Korea

\section{Contents}

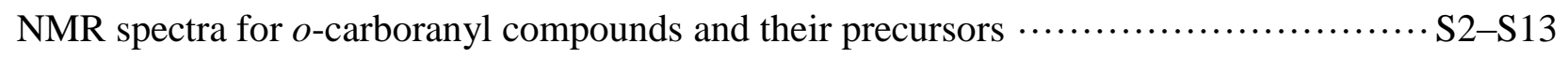

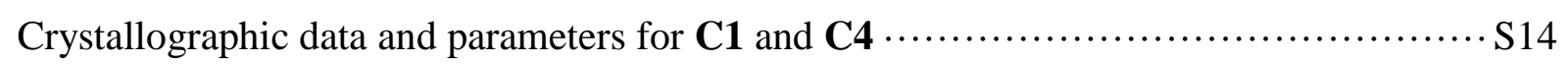

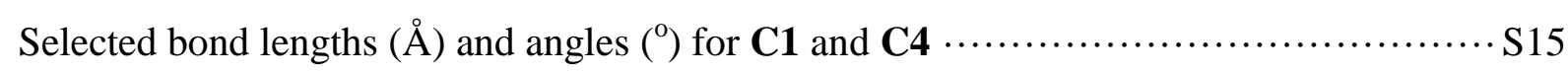

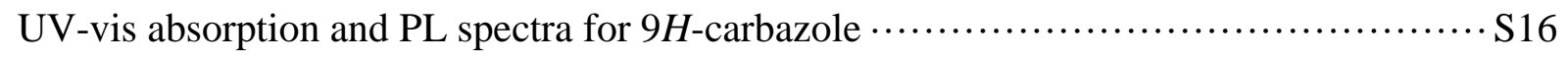

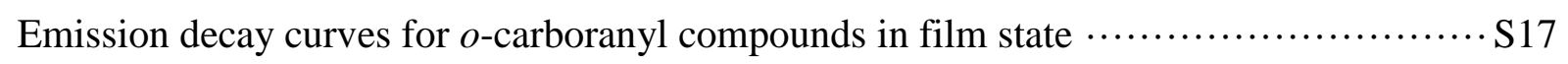

Computational calculation details for $o$-carboranyl compounds …....................S18-S29 


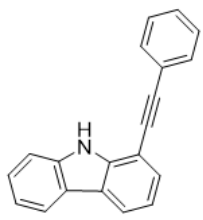

A1

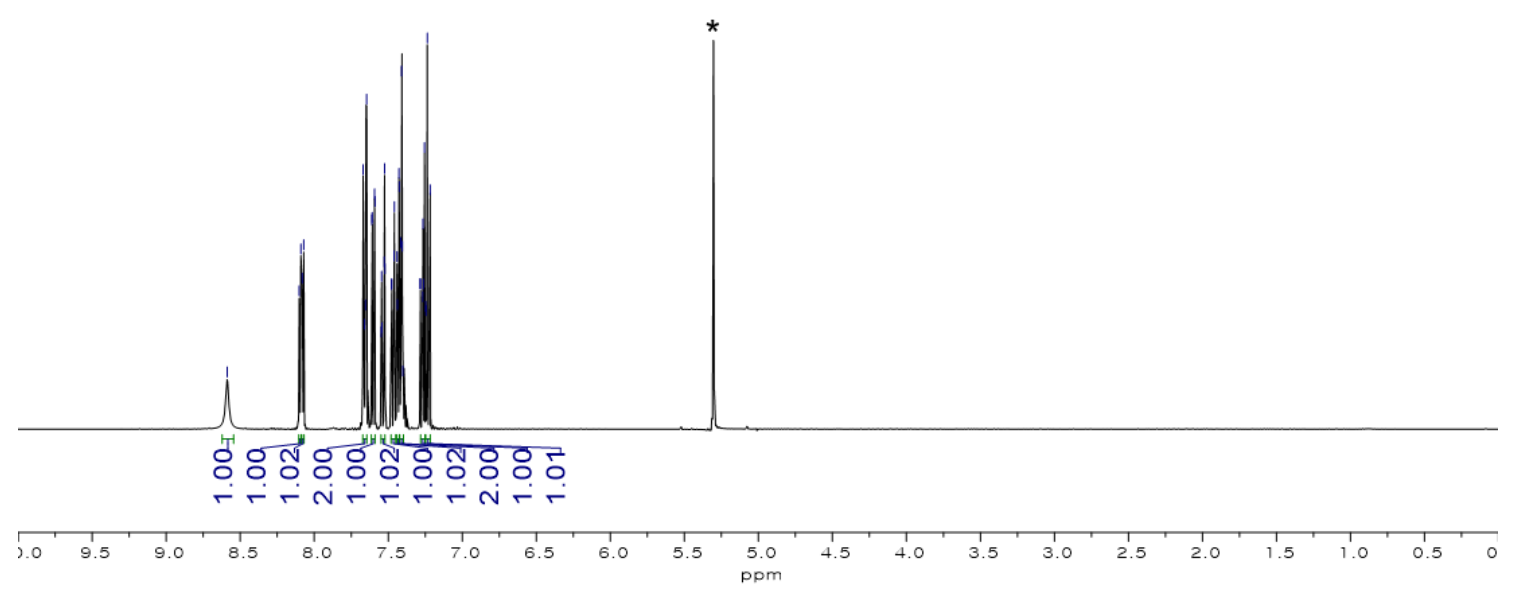

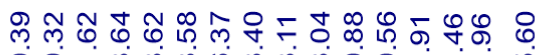

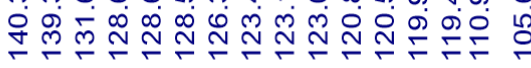

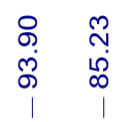
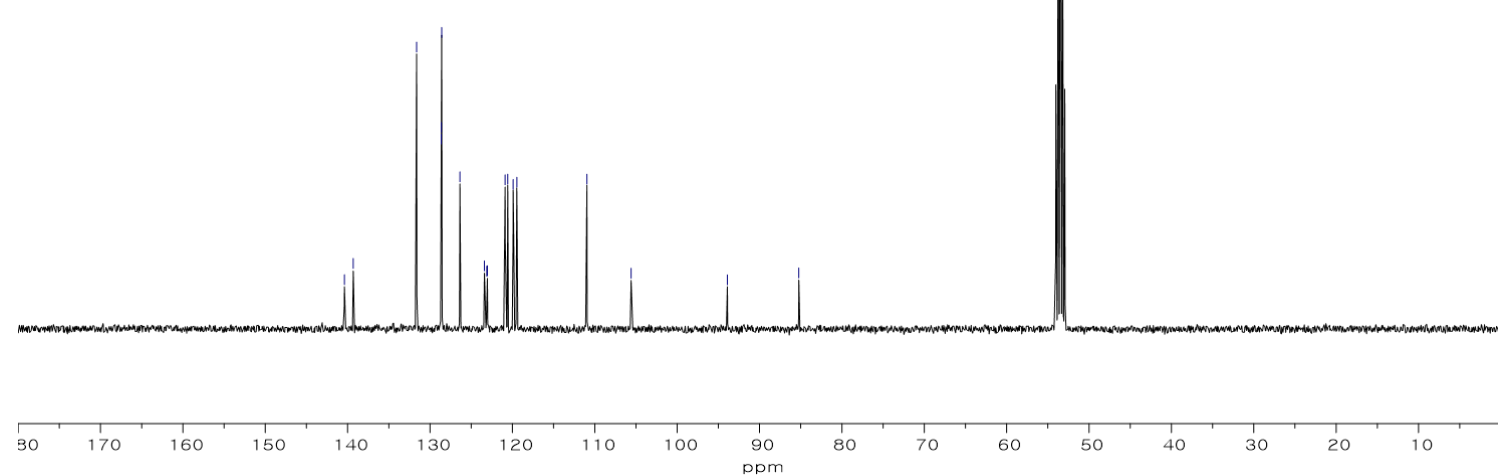

Figure S1. ${ }^{1} \mathrm{H}$ (top) and ${ }^{13} \mathrm{C}$ (bottom) NMR spectra of $\mathbf{A 1}$ in $\mathrm{CD}_{2} \mathrm{Cl}_{2}$ (* from residual $\mathrm{CH}_{2} \mathrm{Cl}_{2}$ in $\mathrm{CD}_{2} \mathrm{Cl}_{2}$ ). 


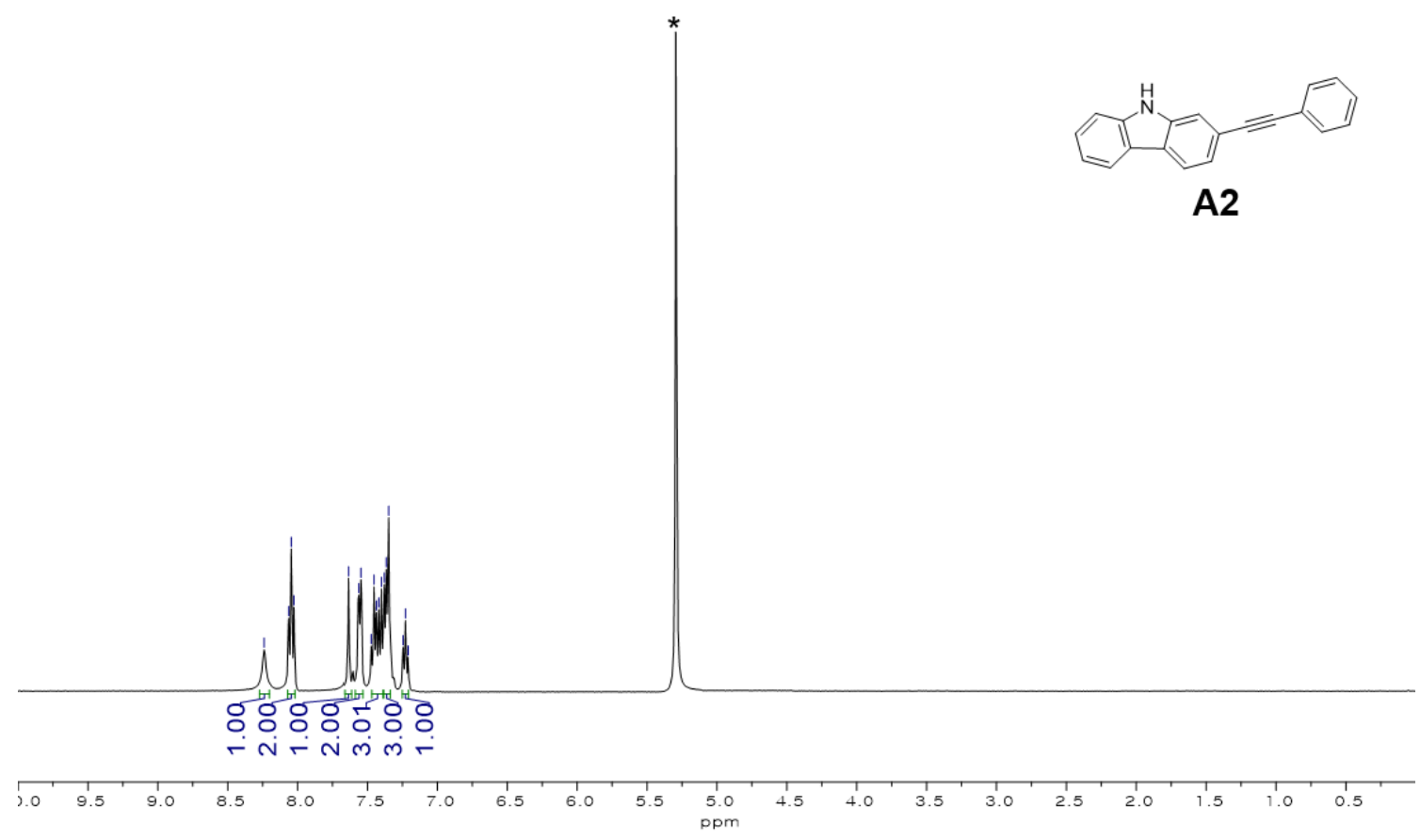

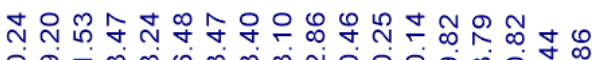

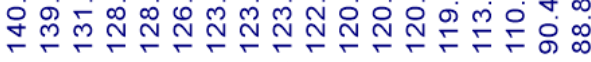
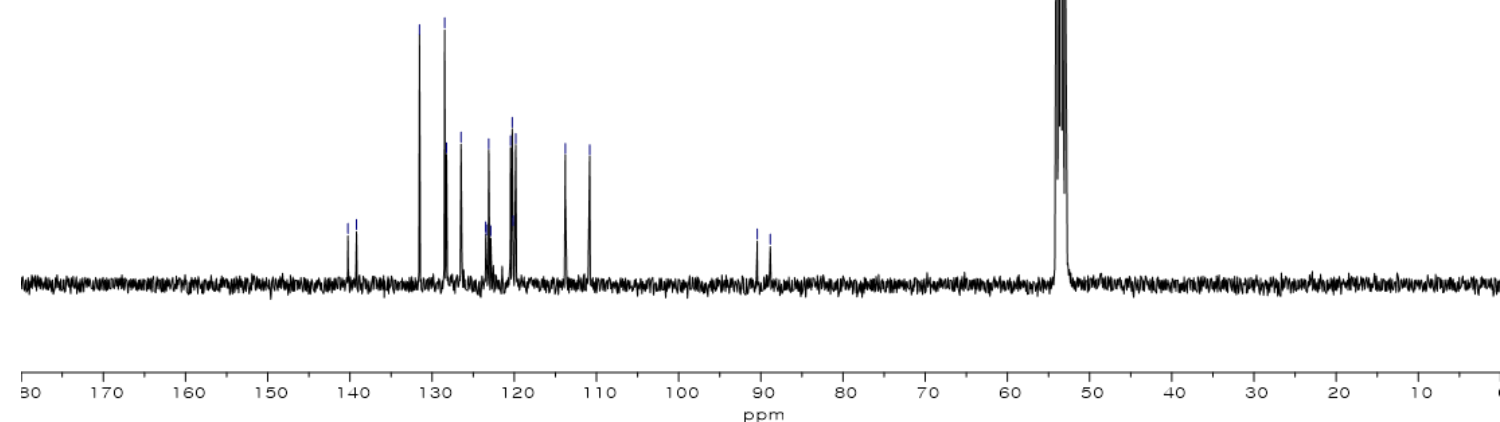

Figure S2. ${ }^{1} \mathrm{H}$ (top) and ${ }^{13} \mathrm{C}$ (bottom) $\mathrm{NMR}$ spectra of $\mathbf{A 2}$ in $\mathrm{CD}_{2} \mathrm{Cl}_{2}$ (* from residual $\mathrm{CH}_{2} \mathrm{Cl}_{2}$ in $\mathrm{CD}_{2} \mathrm{Cl}_{2}$ ). 


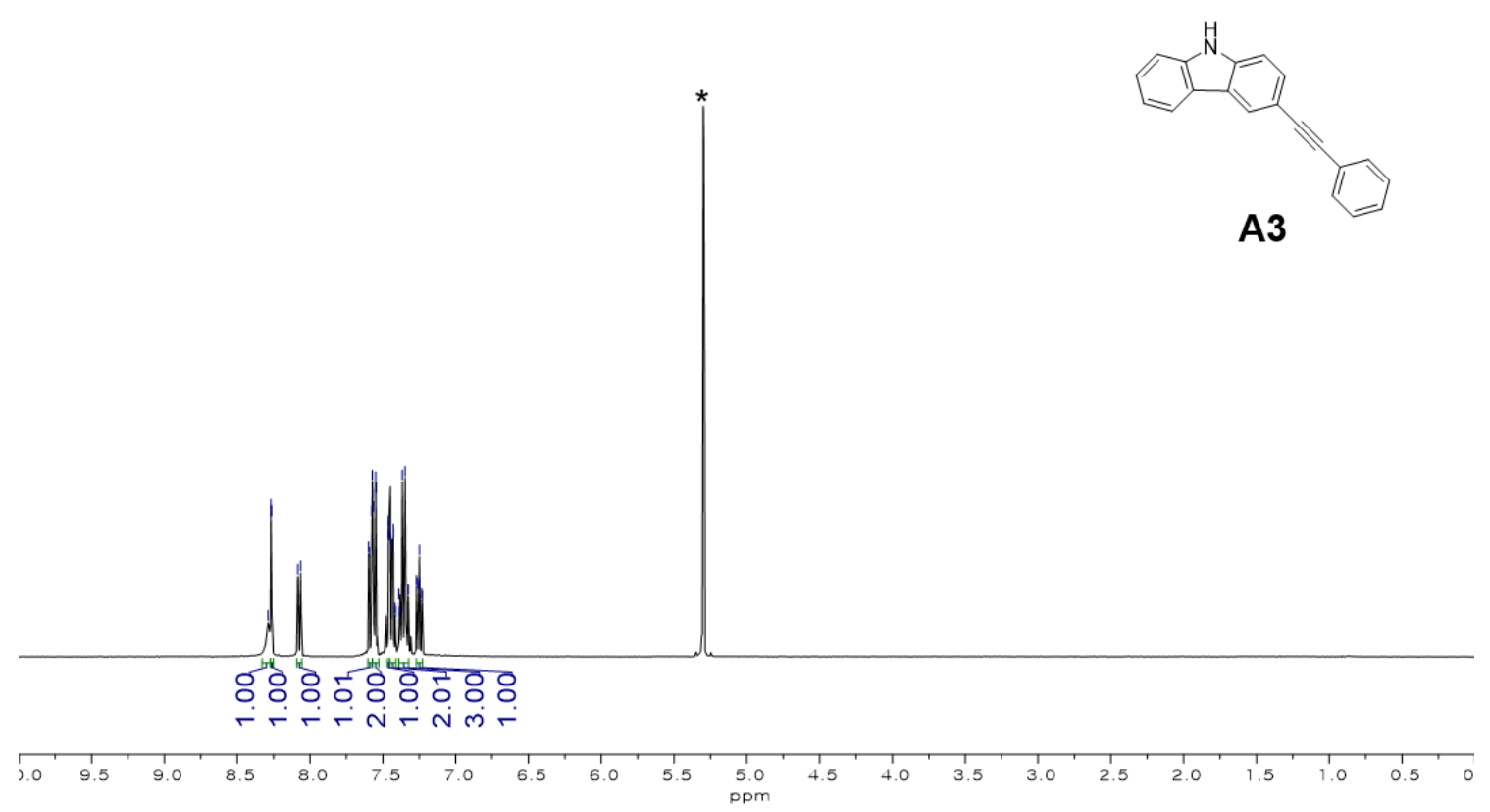

Љำ

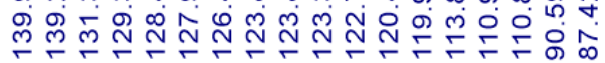
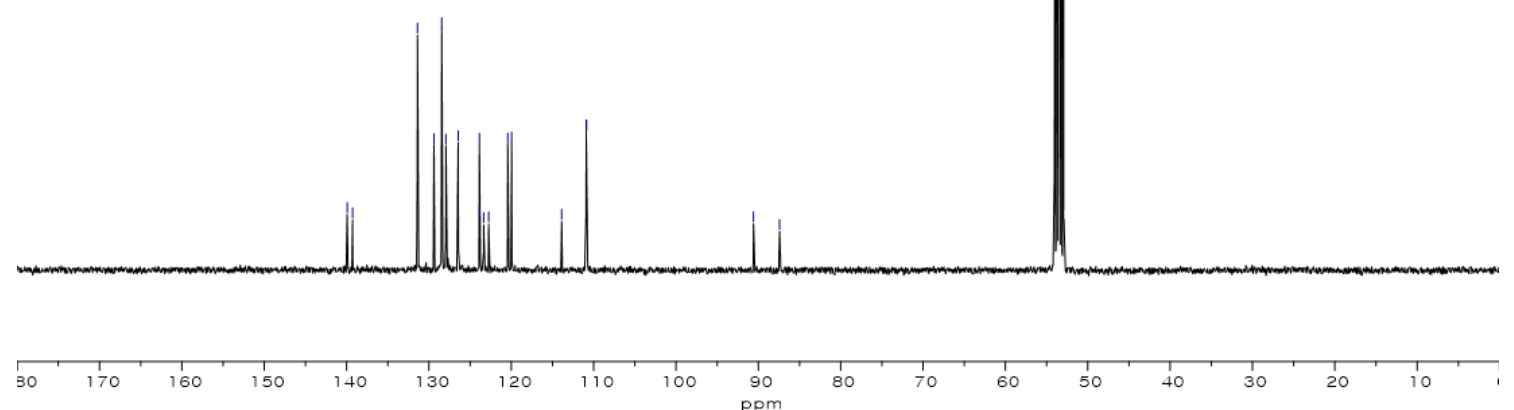

Figure S3. ${ }^{1} \mathrm{H}$ (top) and ${ }^{13} \mathrm{C}$ (bottom) $\mathrm{NMR}$ spectra of $\mathbf{A 3}$ in $\mathrm{CD}_{2} \mathrm{Cl}_{2}$ (* from residual $\mathrm{CH}_{2} \mathrm{Cl}_{2}$ in $\mathrm{CD}_{2} \mathrm{Cl}_{2}$ ). 


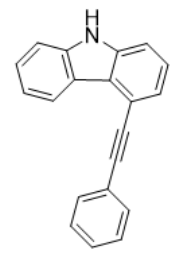

A4

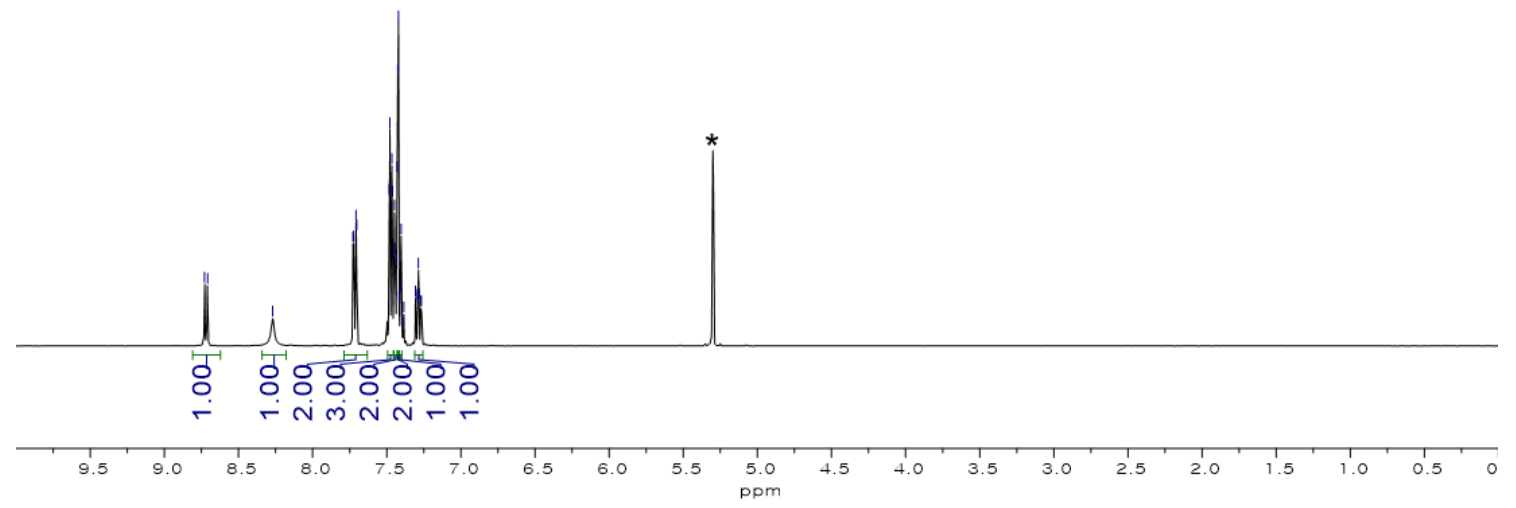

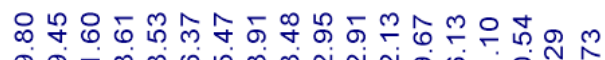

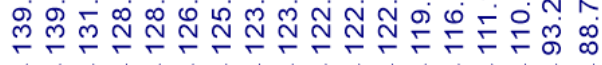
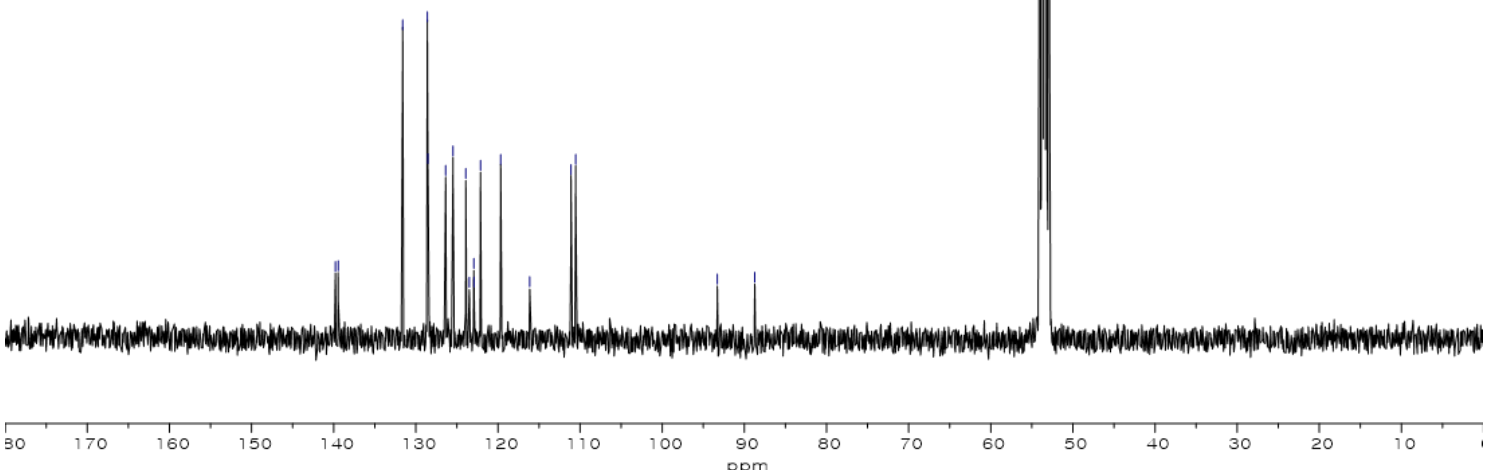

Figure S4. ${ }^{1} \mathrm{H}$ (top) and ${ }^{13} \mathrm{C}$ (bottom) $\mathrm{NMR}$ spectra of $\mathbf{A 4}$ in $\mathrm{CD}_{2} \mathrm{Cl}_{2}$ (* from residual $\mathrm{CH}_{2} \mathrm{Cl}_{2}$ in $\mathrm{CD}_{2} \mathrm{Cl}_{2}$ ). 


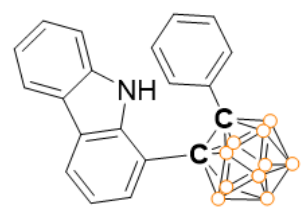

C1 $\triangle \mathrm{BH}$

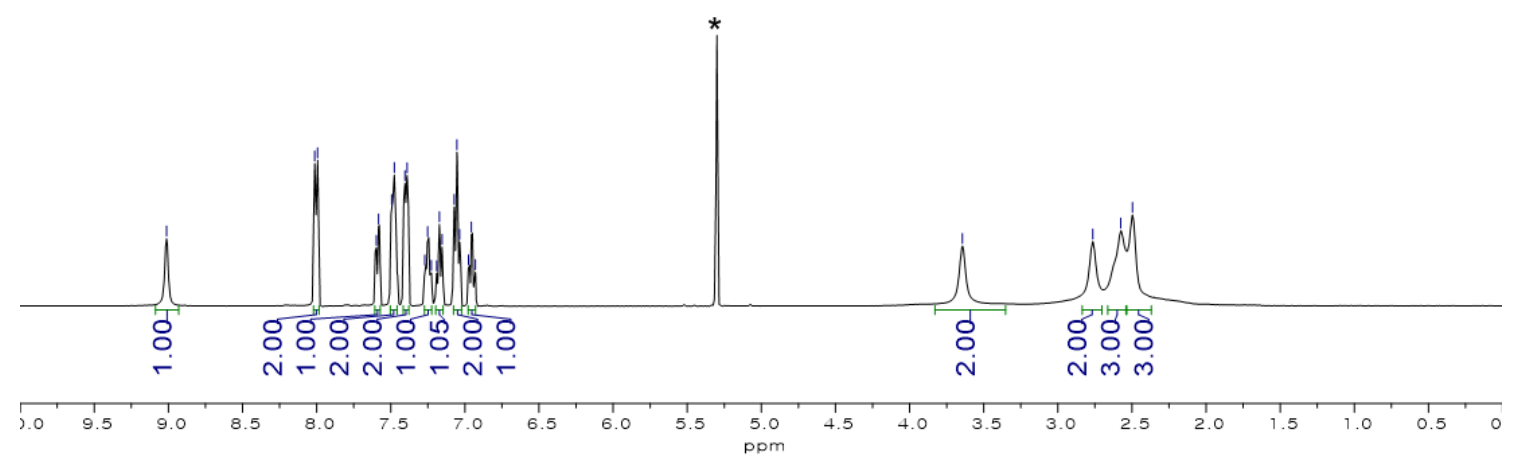

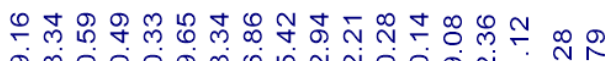

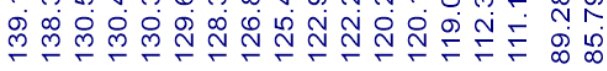
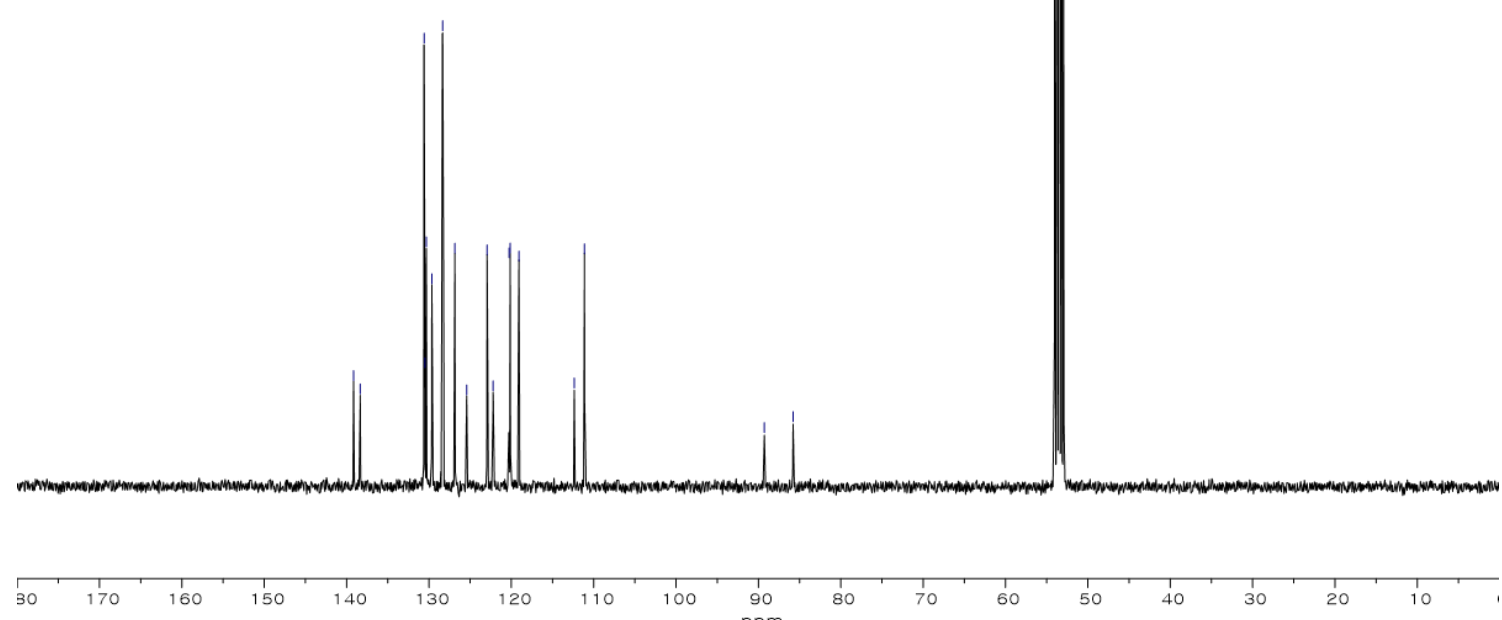

Figure S5. ${ }^{1} \mathrm{H}\left\{{ }^{11} \mathrm{~B}\right\}$ (top) and ${ }^{13} \mathrm{C}$ (bottom) NMR spectra of $\mathbf{C 1}$ in $\mathrm{CD}_{2} \mathrm{Cl}_{2}$ (* from residual $\mathrm{CH}_{2} \mathrm{Cl}_{2}$ in $\mathrm{CD}_{2} \mathrm{Cl}_{2}$ ). 


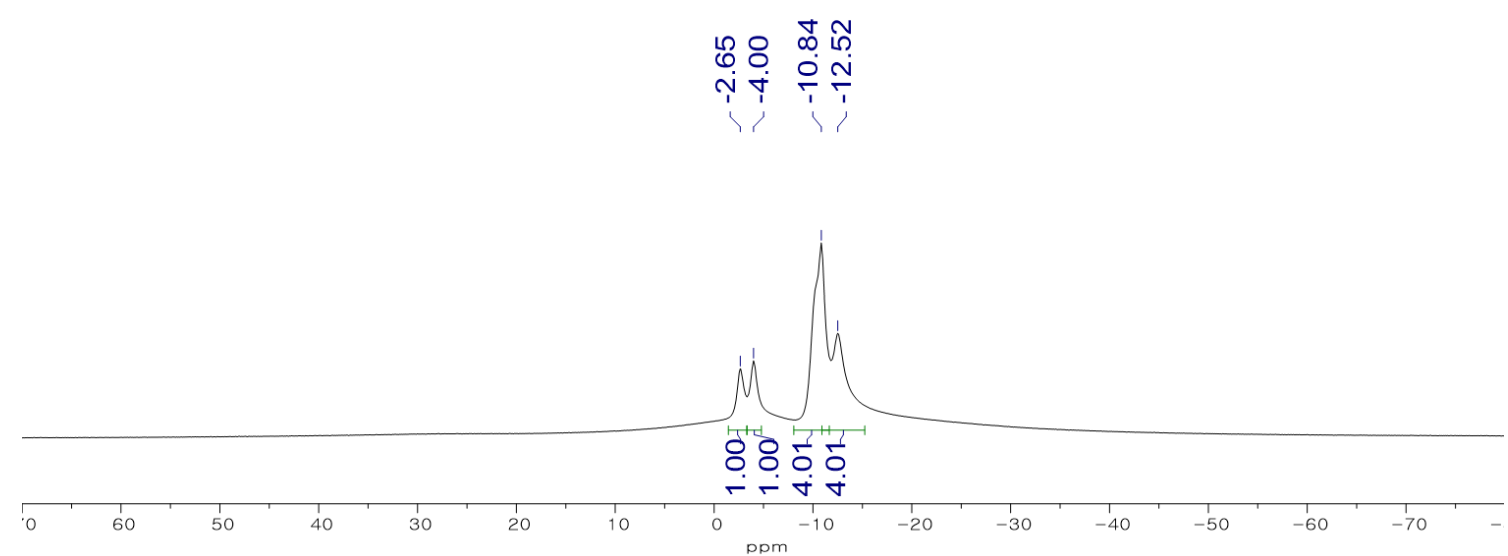

Figure S6. ${ }^{11} \mathrm{~B}\left\{{ }^{1} \mathrm{H}\right\}$ NMR spectra of $\mathbf{C 1}$ in $\mathrm{CD}_{2} \mathrm{Cl}_{2}$. 


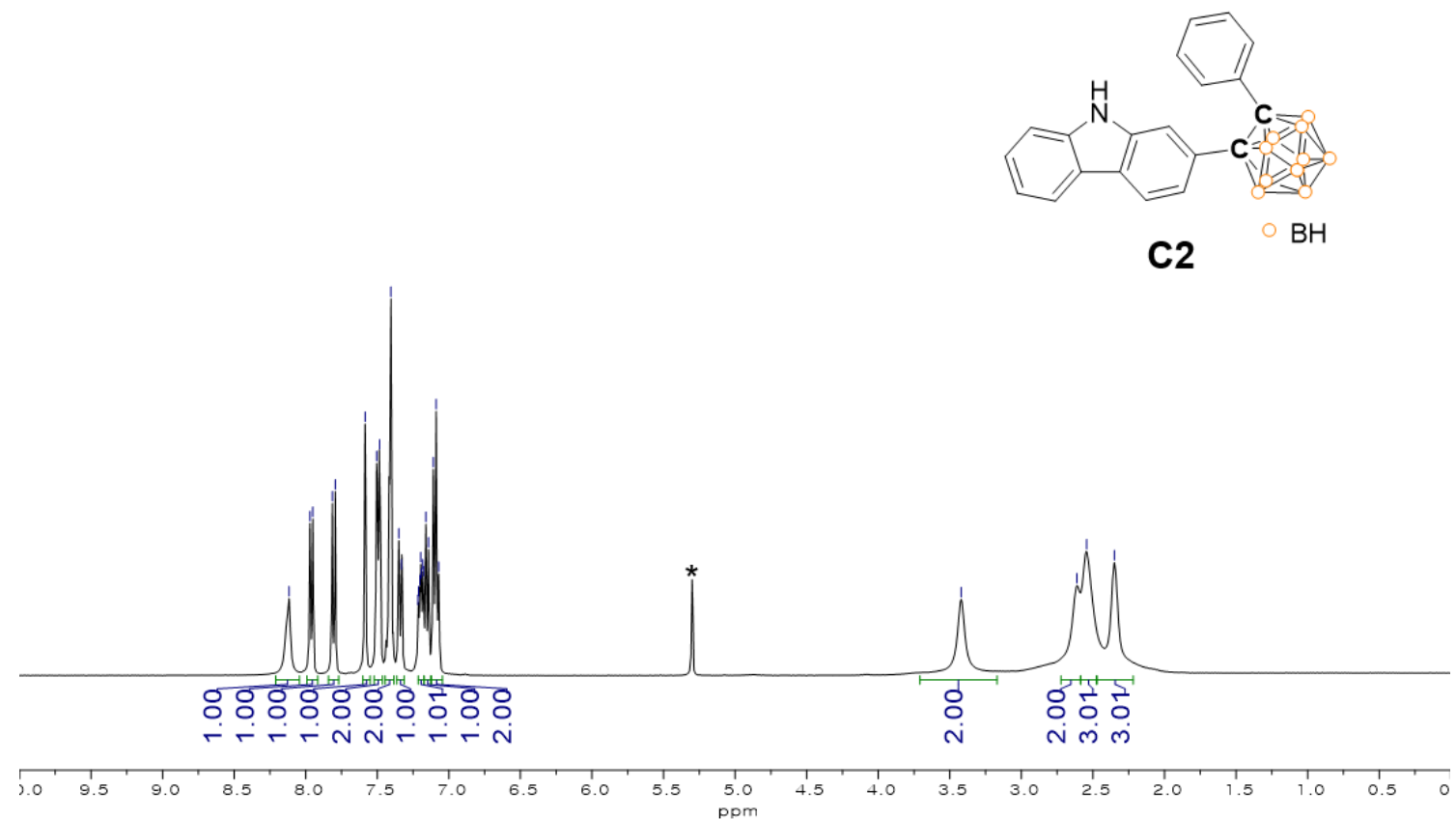

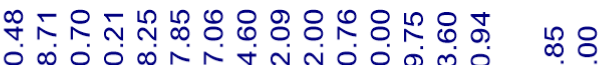

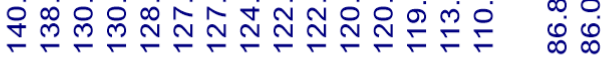
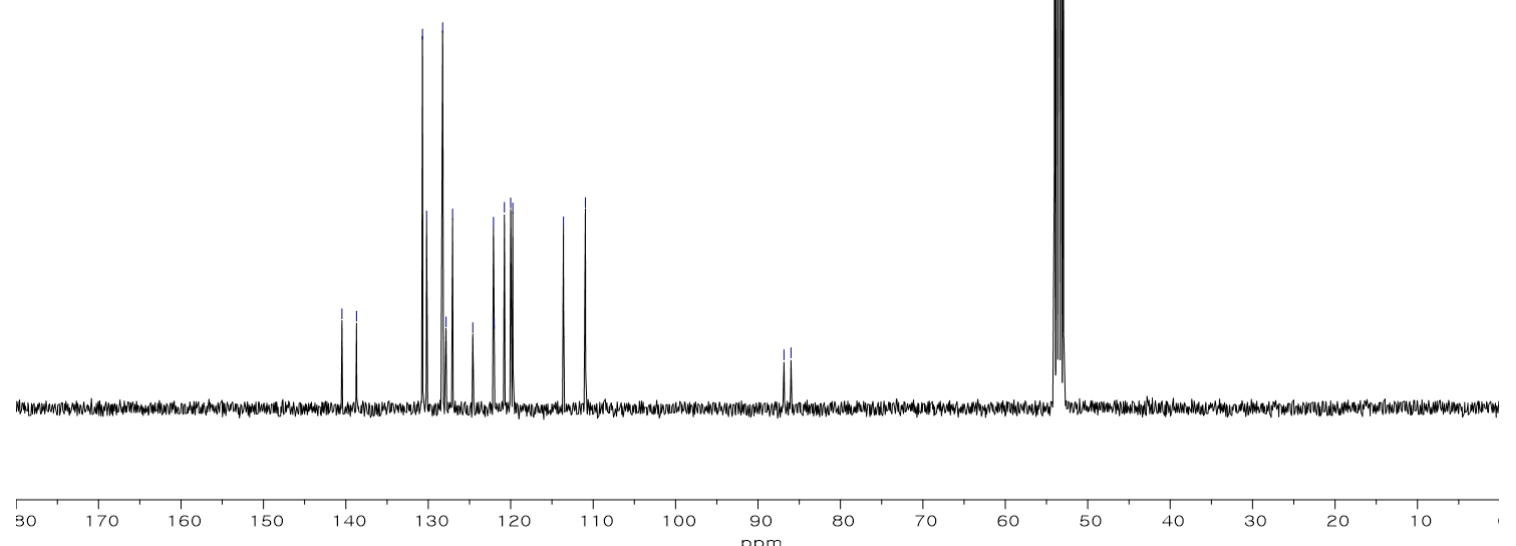

Figure S7. ${ }^{1} \mathrm{H}\left\{{ }^{11} \mathrm{~B}\right\}$ (top) and ${ }^{13} \mathrm{C}$ (bottom) NMR spectra of $\mathbf{C 2}$ in $\mathrm{CD}_{2} \mathrm{Cl}_{2}$ (* from residual $\mathrm{CH}_{2} \mathrm{Cl}_{2}$ in $\mathrm{CD}_{2} \mathrm{Cl}_{2}$ ). 


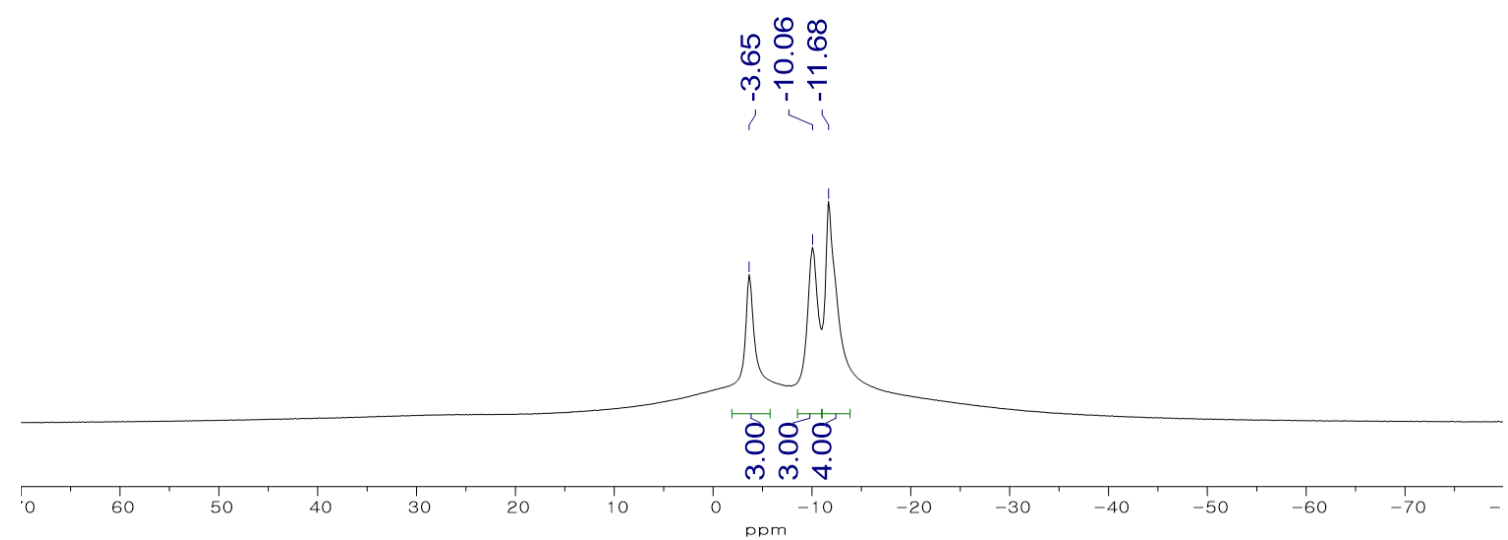

Figure S8. ${ }^{11} \mathrm{~B}\left\{{ }^{1} \mathrm{H}\right\} \mathrm{NMR}$ spectra of $\mathbf{C 2}$ in $\mathrm{CD}_{2} \mathrm{Cl}_{2}$. 

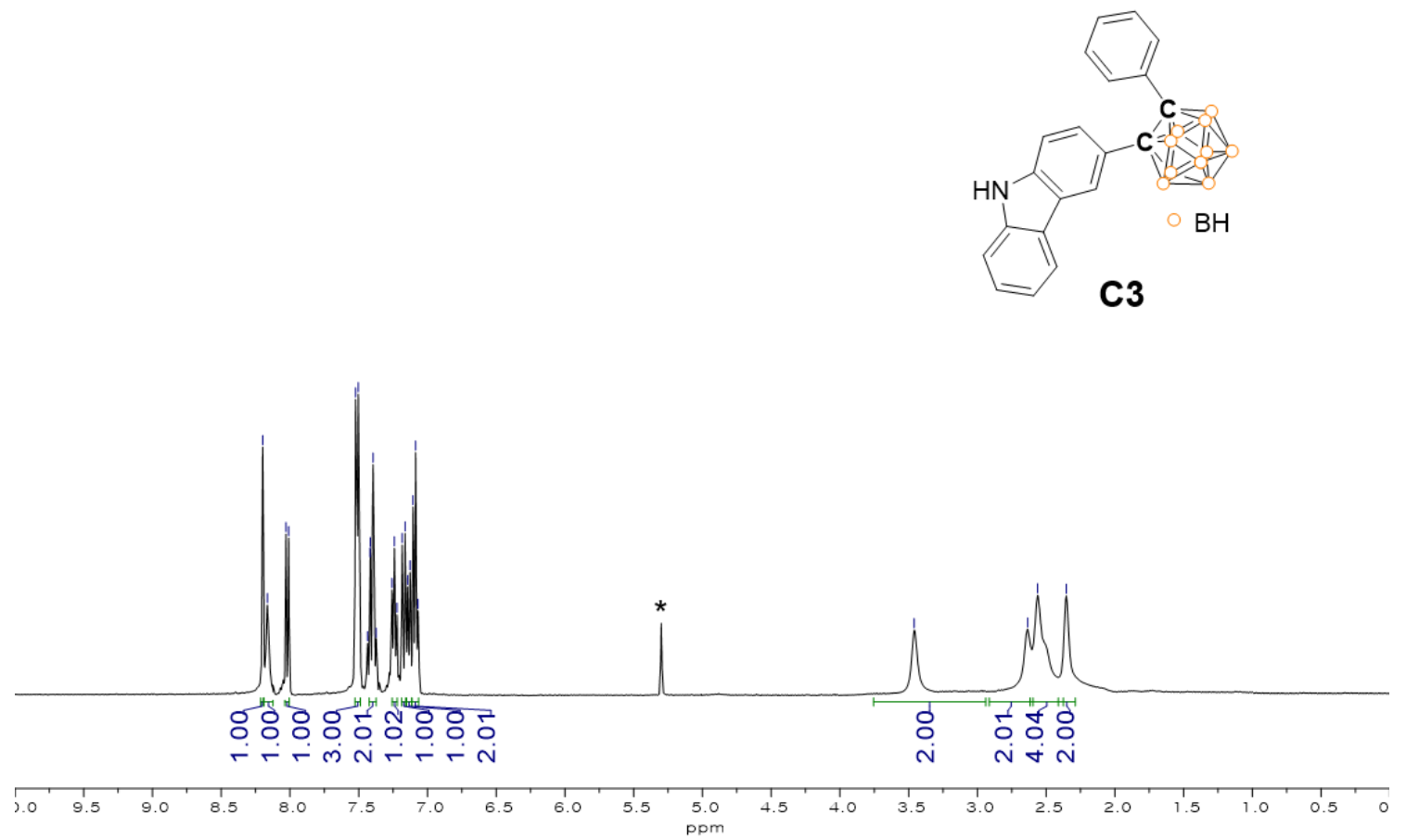

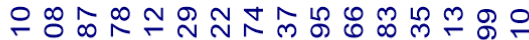

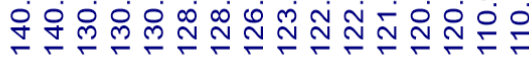

웅ㅇ

ஸे
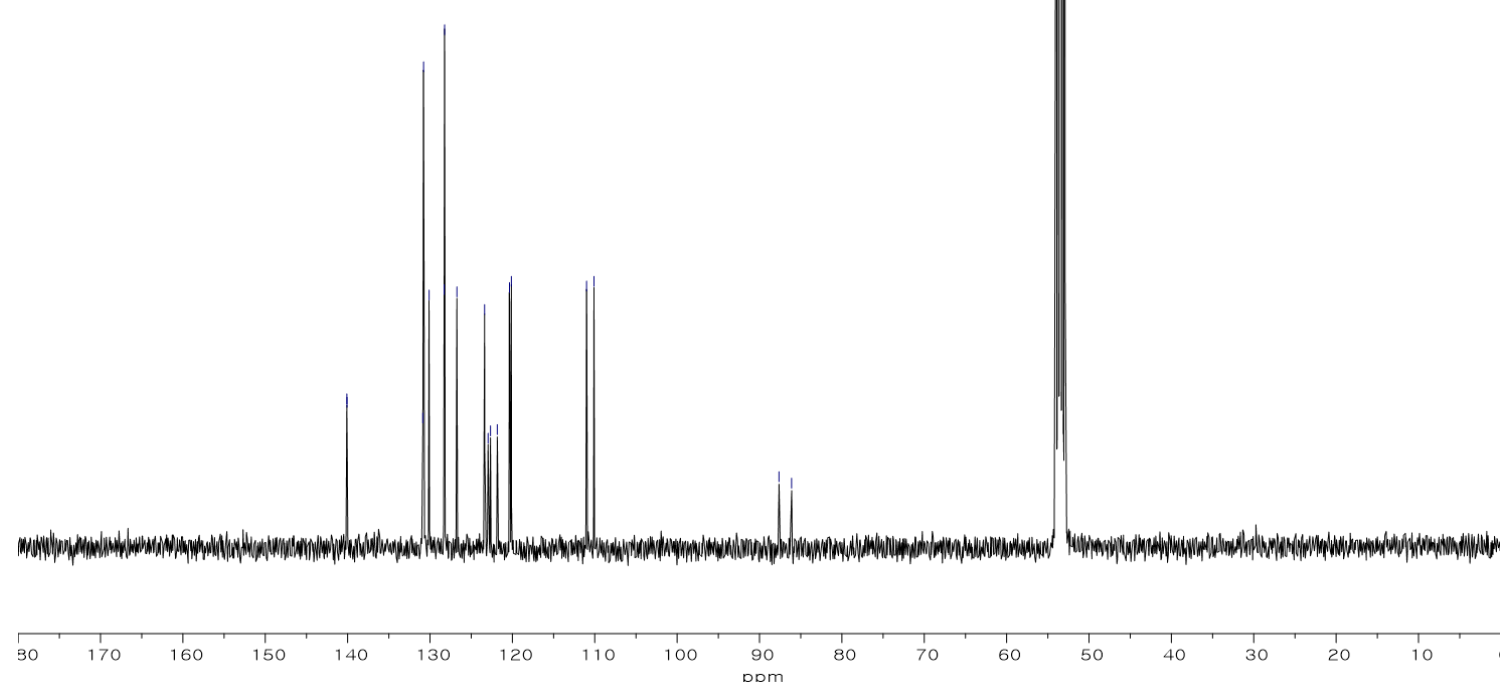

Figure S9. ${ }^{1} \mathrm{H}\left\{{ }^{11} \mathrm{~B}\right\}$ (top) and ${ }^{13} \mathrm{C}$ (bottom) NMR spectra of $\mathbf{C 3}$ in $\mathrm{CD}_{2} \mathrm{Cl}_{2}$ (* from residual $\mathrm{CH}_{2} \mathrm{Cl}_{2}$ in $\mathrm{CD}_{2} \mathrm{Cl}_{2}$ ). 


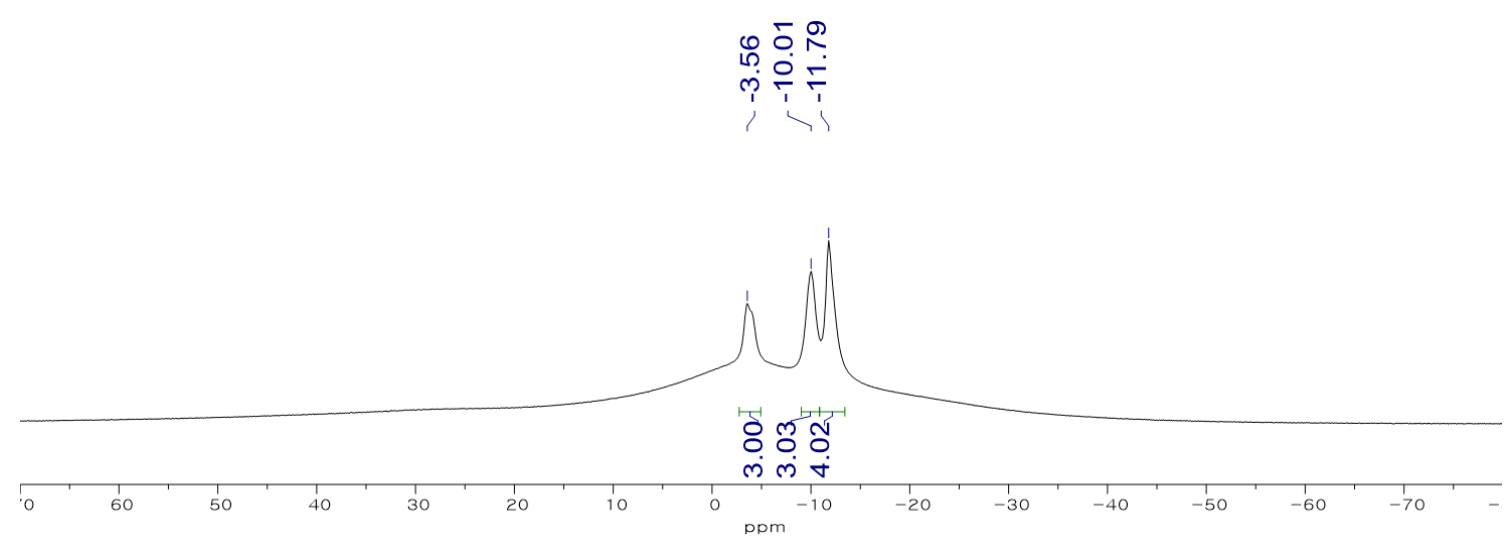

Figure S10. ${ }^{11} \mathrm{~B}\left\{{ }^{1} \mathrm{H}\right\}$ NMR spectra of $\mathbf{C 3}$ in $\mathrm{CD}_{2} \mathrm{Cl}_{2}$. 

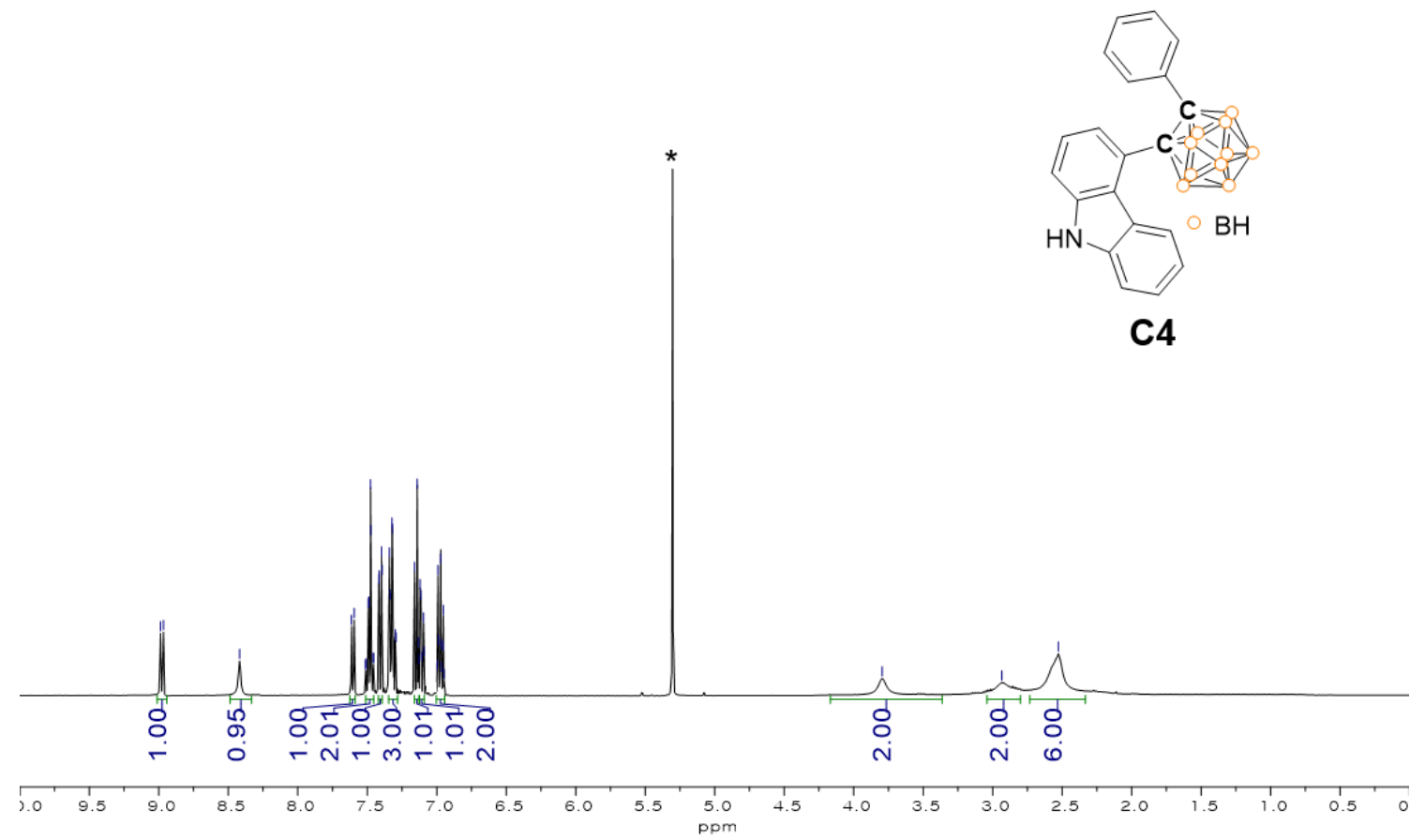

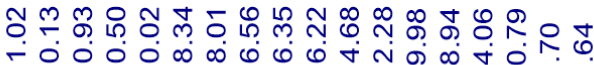

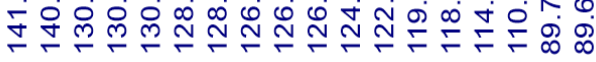

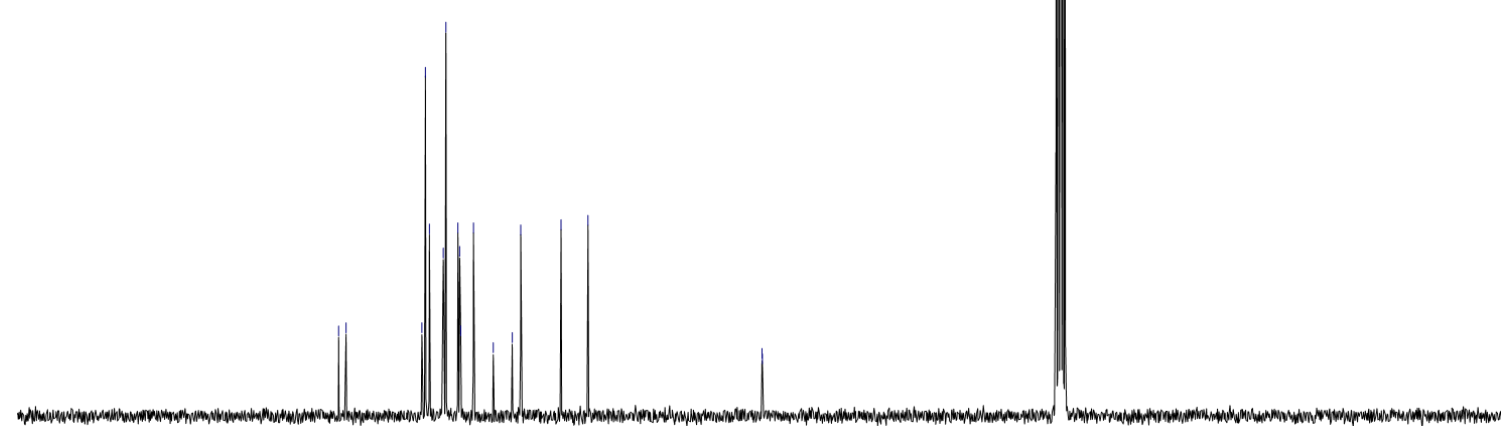

Figure S11. ${ }^{1} \mathrm{H}\left\{{ }^{11} \mathrm{~B}\right\}$ (top) and ${ }^{13} \mathrm{C}$ (bottom) $\mathrm{NMR}$ spectra of $\mathbf{C 4}$ in $\mathrm{CD}_{2} \mathrm{Cl}_{2}$ (* from residual $\mathrm{CH}_{2} \mathrm{Cl}_{2}$ in $\mathrm{CD}_{2} \mathrm{Cl}_{2}$ ). 


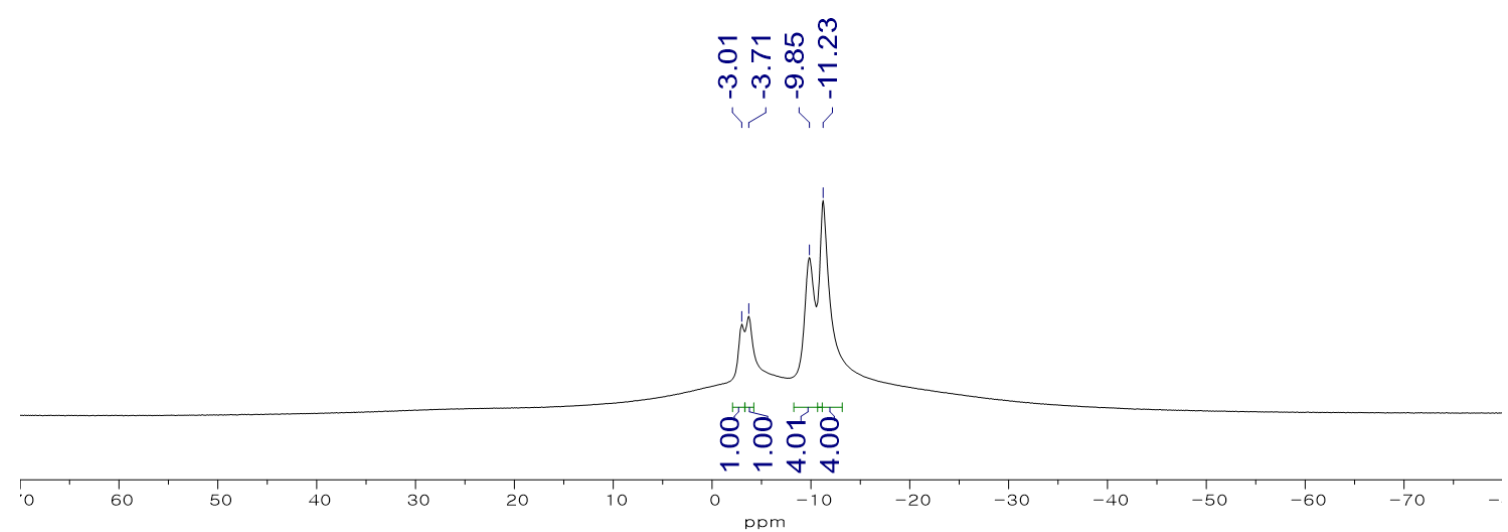

Figure S12. ${ }^{11} \mathrm{~B}\left\{{ }^{1} \mathrm{H}\right\}$ NMR spectra of $\mathbf{C} 4$ in $\mathrm{CD}_{2} \mathrm{Cl}_{2}$. 
Table S1. Crystallographic data and parameters for $\mathbf{C 1}$ and $\mathbf{C 4}$.

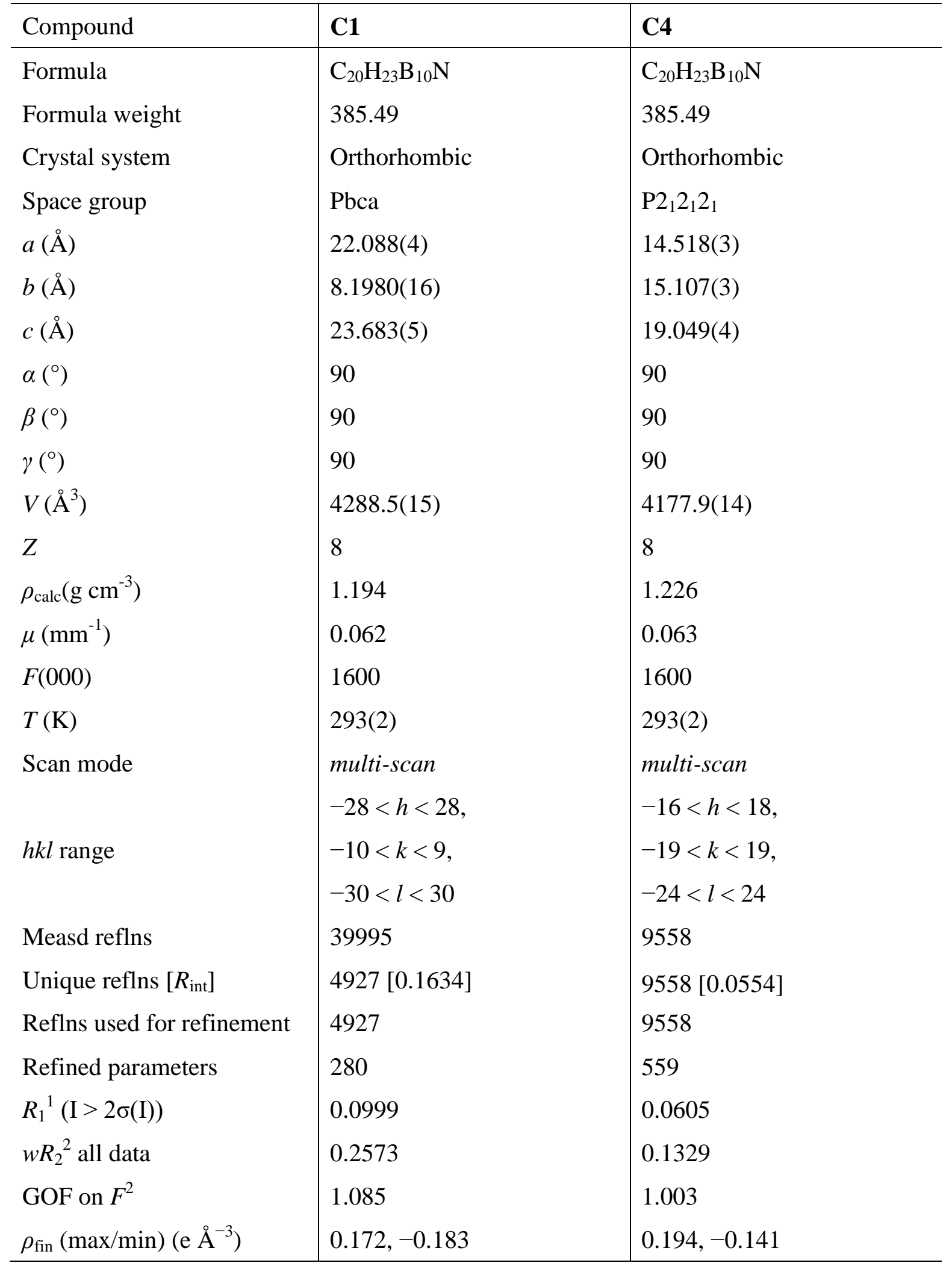

${ }^{1} \mathrm{R}_{1}=\sum|| F_{\mathrm{O}}|-| F \mathrm{c}|| \sum\left|F_{\mathrm{O}}\right| .{ }^{2} w \mathrm{R}_{2}=\left\{\left[\sum w\left(F_{\mathrm{O}}{ }^{2}-F_{\mathrm{c}^{2}}\right)^{2}\right] /\left[\sum w\left(F_{\mathrm{O}}{ }^{2}\right)^{2}\right]\right\}^{1 / 2}$. 
Table S2. Selected bond lengths $(\AA)$ and angles $\left({ }^{\circ}\right)$ for $\mathbf{C 1}$ and $\mathbf{C 4}$.

\begin{tabular}{|c|c|c|}
\hline Compound & C1 & C4 \\
\hline \multicolumn{3}{|c|}{ length $(\AA)$} \\
\hline $\mathrm{C} 1-\mathrm{C} 13$ & $1.508(4)$ & - \\
\hline $\mathrm{C} 4-\mathrm{C} 13$ & - & $1.511(4)$ \\
\hline $\mathrm{C} 13-\mathrm{C} 14$ & $1.753(4)$ & $1.775(5)$ \\
\hline $\mathrm{C} 14-\mathrm{C} 15$ & $1.497(4)$ & $1.502(4)$ \\
\hline \multicolumn{3}{|c|}{ angles $\left(^{\circ}\right)$} \\
\hline $\mathrm{C} 2-\mathrm{C} 1-\mathrm{C} 13$ & $120.2(3)$ & - \\
\hline $\mathrm{C} 3-\mathrm{C} 4-\mathrm{C} 13$ & - & $116.2(3)$ \\
\hline C1-C13-C14 & $120.4(2)$ & - \\
\hline C4-C13-C14 & - & $117.5(2)$ \\
\hline $\mathrm{C} 13-\mathrm{C} 14-\mathrm{C} 15$ & $118.8(2)$ & $118.3(2)$ \\
\hline C9-N1-C12 & $109.6(3)$ & $109.8(3)$ \\
\hline
\end{tabular}




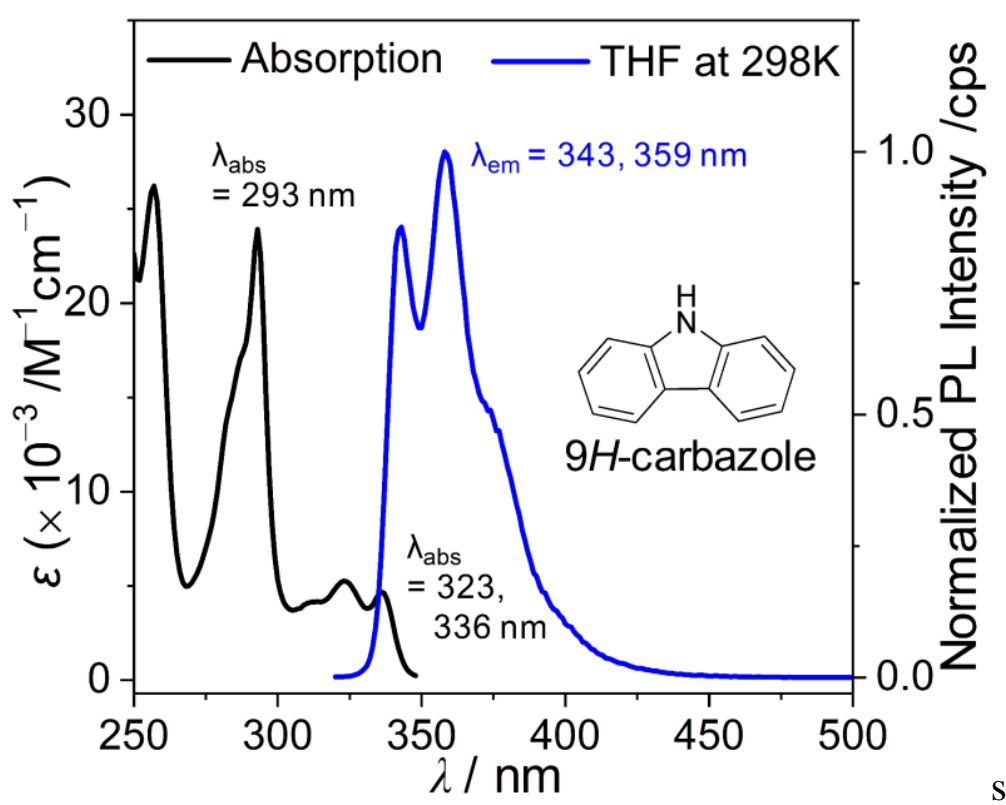

Figure S13. UV-vis absorption (left side) and PL spectra (right side) for $9 H$-carbazole $\left(\lambda_{\mathrm{ex}}=292 \mathrm{~nm}\right.$ ). Black line: absorption spectra in THF $\left(3.0 \times 10^{-5} \mathrm{M}\right)$, blue line: PL spectra in THF $\left(3.0 \times 10^{-5} \mathrm{M}\right)$ at 298 $\mathrm{K}$. 

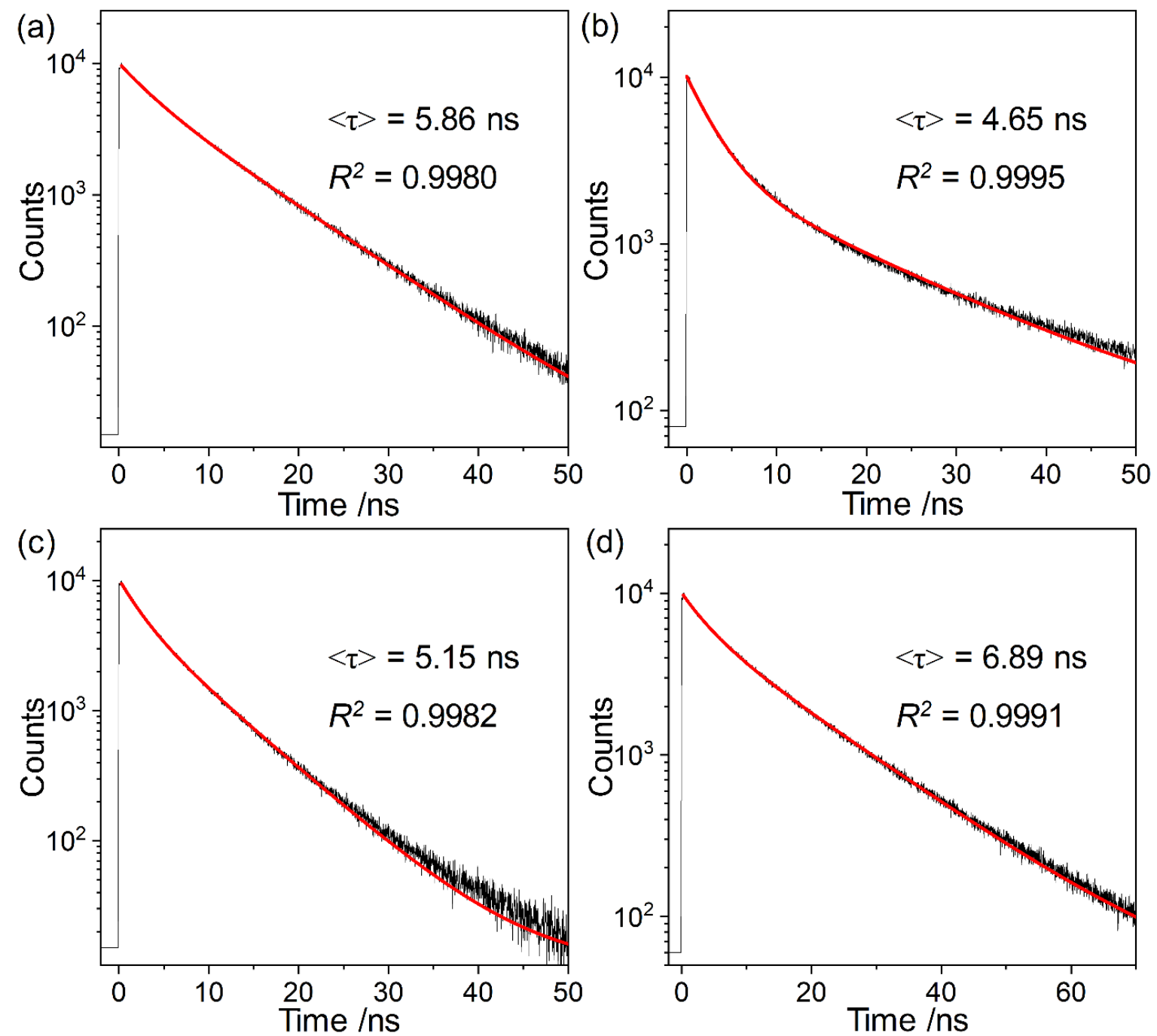

Figure S14. Emission decay curves for (a) C1, (b) C2, (c) C3, and (d) C4 in the film state (5 wt\% doped in PMMA) detected at each CT based emission maxima at $298 \mathrm{~K}$. Each red-line is its single exponential fitting curve for the decay curves. 


\section{Computational calculation details}

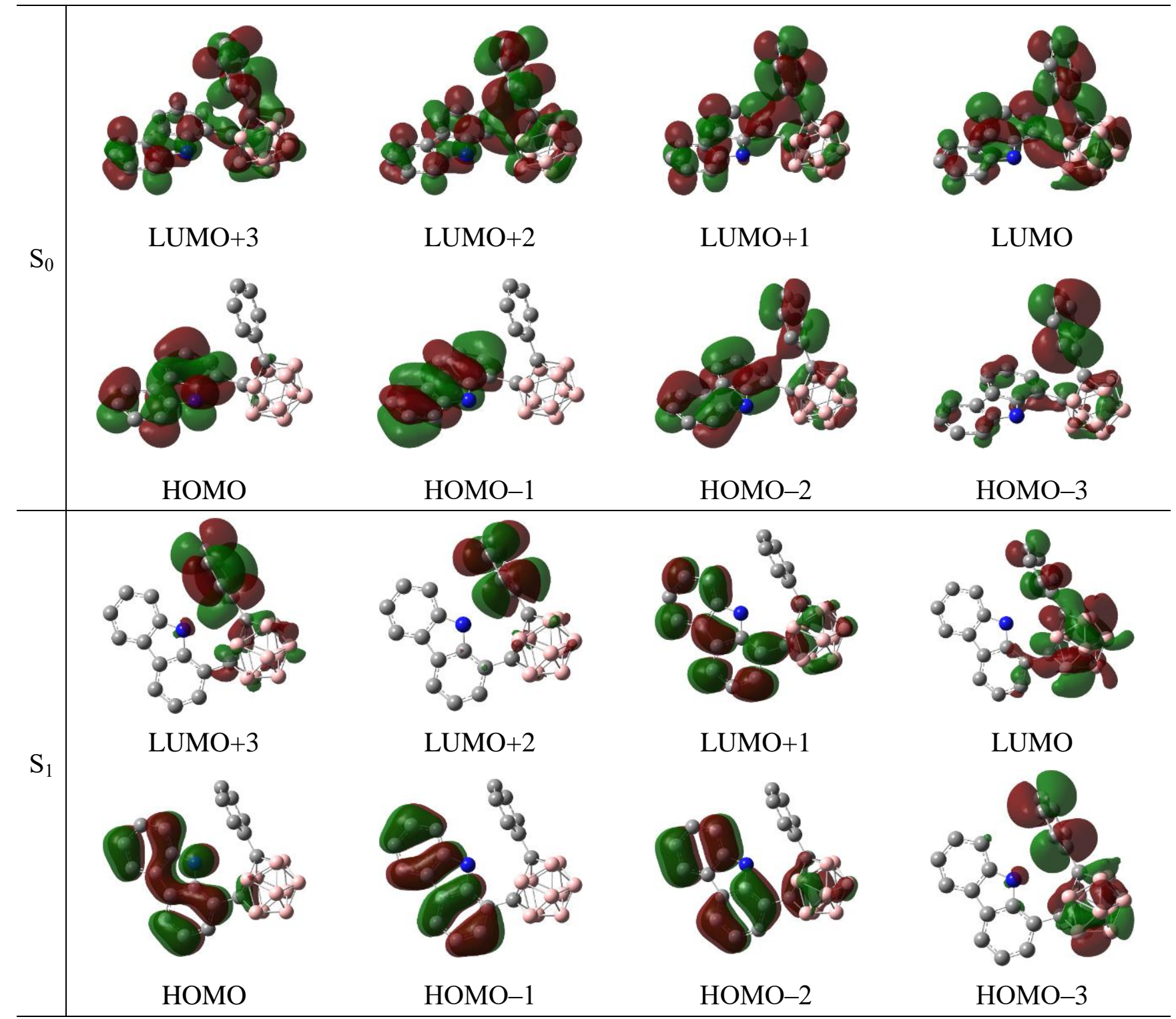

Figure S15. The selected frontier orbitals of C1 from B3LYP calculations (Isovalue $=0.04$ a.u.) at the ground state $\left(S_{0}\right)$ and first singlet excited state $\left(S_{1}\right)$ optimized geometries in THF. 
Table S3. Computed absorption wavelengths $\left(\lambda_{\text {calc }}\right.$ in $\left.\mathrm{nm}\right)$ and oscillator strengths $\left(f_{\text {calc. }}\right)$ for $\mathbf{C 1}$ from TD-B3LYP calculations using the B3LYP geometries at the ground state $\left(\mathrm{S}_{0}\right)$ and first singlet excited state $\left(\mathrm{S}_{1}\right)$ optimized geometries in THF

\begin{tabular}{|c|c|c|c|c|}
\hline state & $\lambda_{\text {calc }}(/ \mathrm{nm})$ & $f_{\text {calc }}$ & \multicolumn{2}{|c|}{ Major contribution } \\
\hline \multicolumn{5}{|c|}{$\mathrm{S}_{0}$} \\
\hline 1 & 343.34 & 0.3208 & HOMO & $\rightarrow \quad$ LUMO $(97.3 \%)$ \\
\hline 2 & 311.17 & 0.0558 & HOMO-1 & $\rightarrow \quad$ LUMO $(93.8 \%)$ \\
\hline 3 & 294.89 & 0.0176 & HOMO & $\rightarrow$ LUMO+1 (91.4\%) \\
\hline \multirow[t]{2}{*}{4} & 273.77 & 0.0537 & HOMO-1 & $\rightarrow$ LUMO+1 (75.9\%) \\
\hline & & & HOMO & $\rightarrow$ LUMO+2 (13.8\%) \\
\hline \multirow[t]{3}{*}{5} & 259.28 & 0.2231 & HOMO-1 & $\rightarrow$ LUMO+1 (11.8\%) \\
\hline & & & HOMO & $\rightarrow$ LUMO+2 $(50.2 \%)$ \\
\hline & & & HOMO & $\rightarrow$ LUMO+3 $(19.5 \%)$ \\
\hline \multicolumn{5}{|c|}{$\mathrm{S}_{1}$} \\
\hline 1 & 584.78 & 0.1987 & HOMO & $\rightarrow$ LUMO (98.8\%) \\
\hline 2 & 457.97 & 0.0020 & HOMO-1 & $\rightarrow \quad$ LUMO $(99.5 \%)$ \\
\hline 3 & 432.88 & 0.0351 & HOMO-2 & $\rightarrow$ LUMO (93.9\%) \\
\hline \multirow[t]{3}{*}{4} & 413.86 & 0.0121 & HOMO-5 & $\rightarrow \quad$ LUMO $(6.6 \%)$ \\
\hline & & & HOMO-4 & $\rightarrow \quad$ LUMO $(18.8 \%)$ \\
\hline & & & HOMO-3 & $\rightarrow \quad$ LUMO $(70.8 \%)$ \\
\hline \multirow[t]{3}{*}{5} & 408.55 & 0.0012 & HOMO-6 & $\rightarrow$ LUMO $(9.7 \%)$ \\
\hline & & & HOMO-4 & $\rightarrow \quad$ LUMO $(60.0 \%)$ \\
\hline & & & HOMO-3 & $\rightarrow \quad$ LUMO $(27.6 \%)$ \\
\hline
\end{tabular}


Table S4. Molecular orbital energies (in eV) and molecular orbital distributions (in \%) of $\mathbf{C 1}$ at the ground state $\left(\mathrm{S}_{0}\right)$ and first singlet excited state $\left(\mathrm{S}_{1}\right)$ optimized geometries in THF

\begin{tabular}{ccccc}
\hline & $\mathrm{E}(\mathrm{eV})$ & $o$-carborane & phenyl & carbazole \\
\hline LUMO+3 & -0.45 & $\mathrm{~S}_{0}$ & & 36.2 \\
LUMO+2 & -0.55 & 19.2 & 44.7 & 32.7 \\
LUMO+1 & -1.03 & 18.7 & 48.6 & 47.8 \\
LUMO & -1.61 & 15.2 & 37.0 & 39.3 \\
HOMO & -5.83 & 40.3 & 20.5 & 97.4 \\
HOMO-1 & -6.10 & 2.4 & 0.1 & 99.2 \\
HOMO-2 & -7.10 & 0.6 & 0.2 & 72.0 \\
HOMO-3 & -7.20 & 6.0 & 22.0 & 8.8 \\
\hline LUMO+3 & -0.77 & 7.4 & 83.8 & \\
LUMO+2 & -0.79 & $\mathrm{~S}_{1}$ & & 2.1 \\
LUMO+1 & -1.04 & 11.7 & 86.2 & 1.0 \\
LUMO & -3.37 & 6.3 & 92.7 & 87.8 \\
HOMO & -5.56 & 11.5 & 0.7 & 2.4 \\
HOMO-1 & -6.07 & 84.2 & 13.4 & 96.3 \\
HOMO-2 & -6.85 & 3.5 & 0.2 & 9.2 \\
HOMO-3 & -7.46 & 0.8 & 0.0 & 8.9 \\
\hline
\end{tabular}




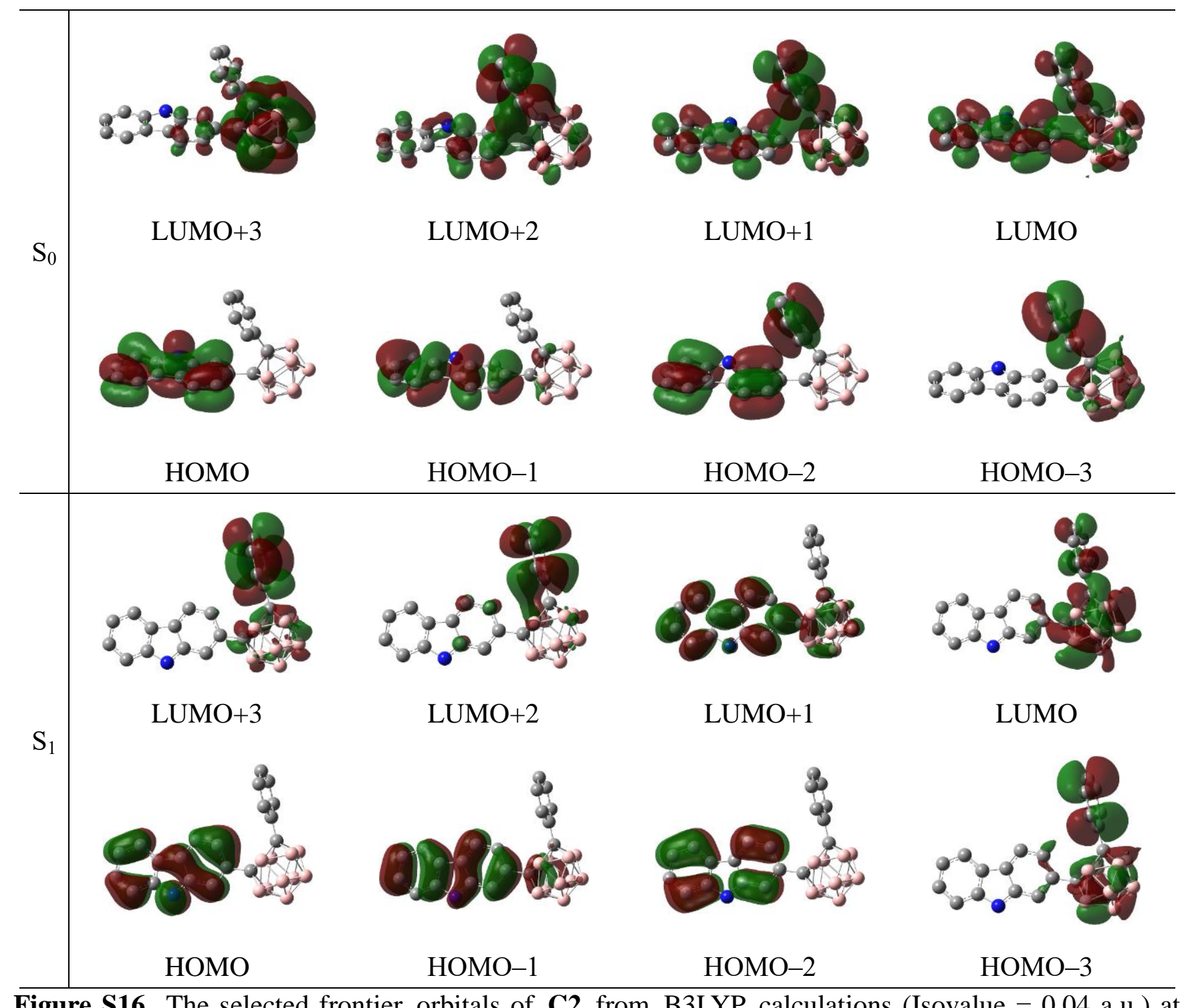

Figure S16. The selected frontier orbitals of C2 from B3LYP calculations (Isovalue $=0.04$ a.u.) at the ground state $\left(S_{0}\right)$ and first singlet excited state $\left(S_{1}\right)$ optimized geometries in THF. 
Table S5. Computed absorption wavelengths $\left(\lambda_{\text {calc }}\right.$ in $\left.\mathrm{nm}\right)$ and oscillator strengths $\left(f_{\text {calc. }}\right)$ for $\mathbf{C 2}$ from TD-B3LYP calculations using the B3LYP geometries at the ground state $\left(\mathrm{S}_{0}\right)$ and first singlet excited state $\left(\mathrm{S}_{1}\right)$ optimized geometries in THF

\begin{tabular}{|c|c|c|c|c|}
\hline state & $\lambda_{\text {calc }}(/ \mathrm{nm})$ & $f_{\text {calc }}$ & \multicolumn{2}{|c|}{ Major contribution } \\
\hline \multicolumn{5}{|c|}{$\mathrm{S}_{0}$} \\
\hline 1 & 335.71 & 0.2299 & HOMO & $\rightarrow \quad$ LUMO $(95.1 \%)$ \\
\hline 2 & 303.83 & 0.0684 & HOMO-1 & $\rightarrow \quad$ LUMO $(89.6 \%)$ \\
\hline 3 & 291.91 & 0.0512 & HOMO & $\rightarrow$ LUMO+1 $(90.7 \%)$ \\
\hline \multirow[t]{2}{*}{4} & 271.46 & 0.0089 & HOMO-1 & $\rightarrow$ LUMO+1 $(78.5 \%)$ \\
\hline & & & HOMO & $\rightarrow$ LUMO+2 (11.7\%) \\
\hline \multirow[t]{2}{*}{5} & 259.46 & 0.2032 & HOMO-1 & $\rightarrow$ LUMO+1 $(10.2 \%)$ \\
\hline & & & HOMO & $\rightarrow$ LUMO+2 $(84.8 \%)$ \\
\hline \multicolumn{5}{|c|}{$\mathrm{S}_{1}$} \\
\hline 1 & 604.99 & 0.2208 & HOMO & $\rightarrow \quad$ LUMO $(98.2 \%)$ \\
\hline 2 & 499.60 & 0.0008 & HOMO-1 & $\rightarrow \quad$ LUMO $(96.6 \%)$ \\
\hline 3 & 430.98 & 0.0002 & HOMO-2 & $\rightarrow \quad$ LUMO $(98.4 \%)$ \\
\hline \multirow[t]{2}{*}{4} & 410.58 & 0.0019 & HOMO-4 & $\rightarrow \quad$ LUMO $(22.6 \%)$ \\
\hline & & & HOMO-3 & $\rightarrow \quad$ LUMO $(69.9 \%)$ \\
\hline \multirow[t]{3}{*}{5} & 403.60 & 0.0024 & HOMO-5 & $\rightarrow \quad \operatorname{LUMO}(8.5 \%)$ \\
\hline & & & HOMO-4 & $\rightarrow \quad$ LUMO $(59.6 \%)$ \\
\hline & & & HOMO-3 & $\rightarrow$ LUMO (29.1\%) \\
\hline
\end{tabular}


Table S6. Molecular orbital energies (in eV) and molecular orbital distributions (in \%) of $\mathbf{C 2}$ at the ground state $\left(\mathrm{S}_{0}\right)$ and first singlet excited state $\left(\mathrm{S}_{1}\right)$ optimized geometries in THF

\begin{tabular}{ccccc}
\hline & $\mathrm{E}(\mathrm{eV})$ & $o$-carborane & phenyl & carbazole \\
\hline LUMO+3 & -0.24 & $\mathrm{~S}_{0}$ & 3.2 & 6.2 \\
LUMO+2 & -0.56 & 11.8 & 69.8 & 18.3 \\
LUMO+1 & -1.00 & 17.6 & 48.6 & 33.8 \\
LUMO & -1.54 & 32.6 & 14.6 & 52.9 \\
HOMO & -5.78 & 0.3 & 0.1 & 99.6 \\
HOMO-1 & -6.11 & 4.1 & 0.2 & 95.7 \\
HOMO-2 & -7.11 & 0.5 & 29.1 & 70.4 \\
HOMO-3 & -7.15 & 11.4 & 87.8 & 0.8 \\
\hline LUMO+3 & -0.48 & $\mathrm{~S}_{1}$ & & \\
LUMO+2 & -0.61 & 12.6 & 86.8 & 0.6 \\
LUMO+1 & -1.25 & 7.8 & 87.9 & 4.3 \\
LUMO & -3.35 & 11.9 & 0.1 & 88.0 \\
HOMO & -5.57 & 83.4 & 14.5 & 2.1 \\
HOMO-1 & -6.00 & 1.0 & 0.0 & 99.0 \\
HOMO-2 & -7.00 & 5.9 & 0.0 & 9.0 \\
HOMO-3 & -7.25 & 0.4 & 0.2 & 9.4 \\
\hline
\end{tabular}




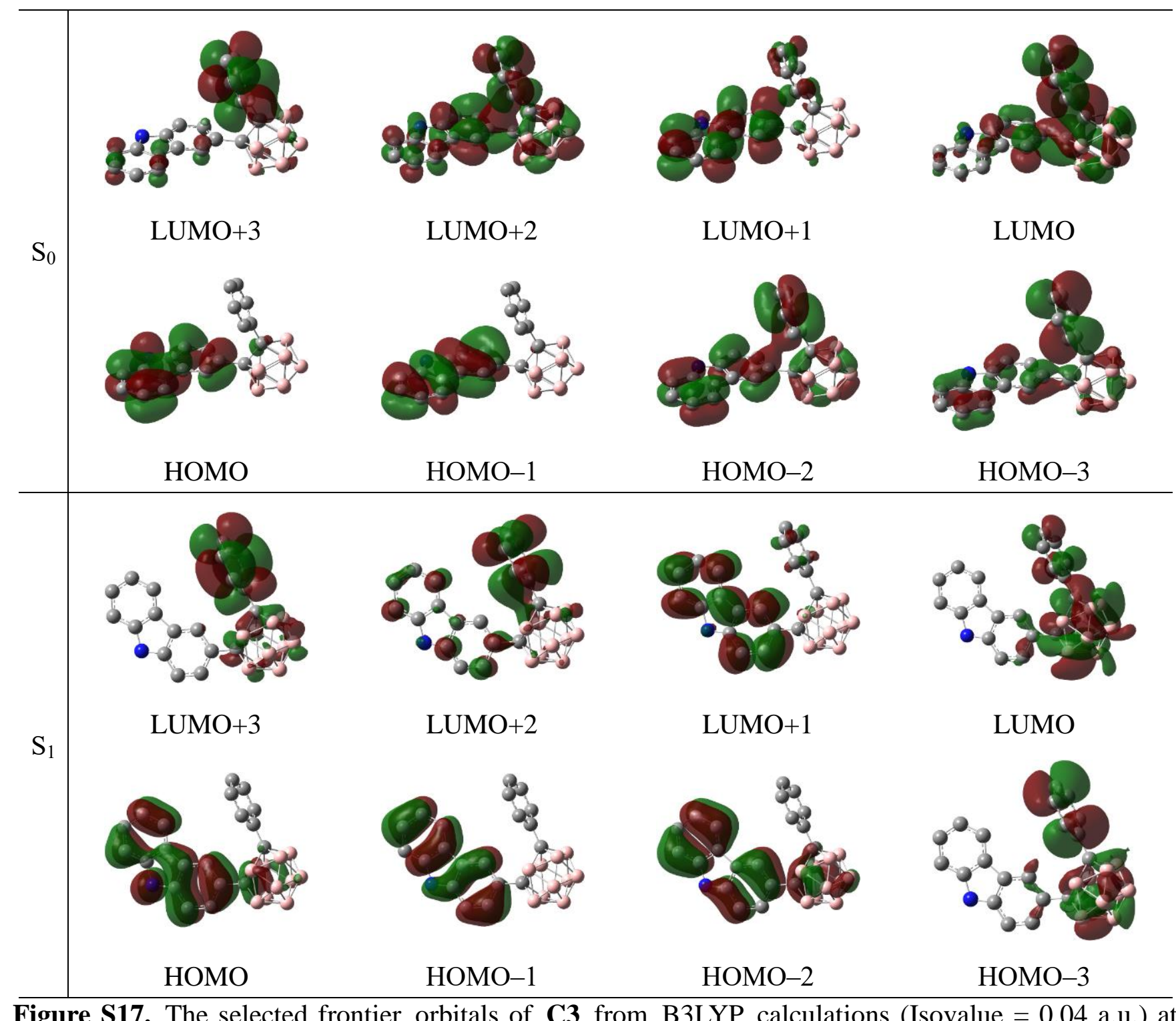

Figure S17. The selected frontier orbitals of C3 from B3LYP calculations (Isovalue $=0.04$ a.u.) at the ground state $\left(\mathrm{S}_{0}\right)$ and first singlet excited state $\left(\mathrm{S}_{1}\right)$ optimized geometries in THF. 
Table S7. Computed absorption wavelengths $\left(\lambda_{\text {calc }}\right.$ in $\left.\mathrm{nm}\right)$ and oscillator strengths $\left(f_{\text {calc. }}\right)$ for $\mathbf{C 3}$ from TD-B3LYP calculations using the B3LYP geometries at the ground state $\left(\mathrm{S}_{0}\right)$ and first singlet excited state $\left(\mathrm{S}_{1}\right)$ optimized geometries in THF

\begin{tabular}{|c|c|c|c|c|}
\hline state & $\lambda_{\text {calc }}(/ \mathrm{nm})$ & $f_{\text {calc }}$ & \multicolumn{2}{|c|}{ Major contribution } \\
\hline \multicolumn{5}{|c|}{$\mathrm{S}_{0}$} \\
\hline \multirow[t]{2}{*}{1} & 324.56 & 0.2006 & HOMO & $\rightarrow \quad$ LUMO $(88.4 \%)$ \\
\hline & & & HOMO & $\rightarrow$ LUMO+1 (6.6\%) \\
\hline \multirow[t]{2}{*}{2} & 306.72 & 0.0242 & HOMO-1 & $\rightarrow \quad$ LUMO (20.7\%) \\
\hline & & & HOMO & $\rightarrow$ LUMO+1 $(65.0 \%)$ \\
\hline \multirow[t]{2}{*}{3} & 289.53 & 0.0548 & HOMO-1 & $\rightarrow \quad$ LUMO $(71.6 \%)$ \\
\hline & & & HOMO & $\rightarrow$ LUMO+1 (19.6\%) \\
\hline \multirow[t]{2}{*}{4} & 276.73 & 0.0811 & HOMO-1 & $\rightarrow$ LUMO+1 $(58.6 \%)$ \\
\hline & & & HOMO & $\rightarrow$ LUMO+2 (29.6\%) \\
\hline \multirow[t]{2}{*}{5} & 257.18 & 0.1787 & HOMO-1 & $\rightarrow$ LUMO+1 $(27.7 \%)$ \\
\hline & & & HOMO & $\rightarrow$ LUMO+2 $(60.7 \%)$ \\
\hline \multicolumn{5}{|c|}{$\mathrm{S}_{1}$} \\
\hline 1 & 564.12 & 0.1826 & HOMO & $\rightarrow \quad$ LUMO $(97.9 \%)$ \\
\hline 2 & 519.89 & 0.0005 & HOMO-1 & $\rightarrow \quad$ LUMO $(99.1 \%)$ \\
\hline 3 & 473.10 & 0.0028 & HOMO-2 & $\rightarrow \quad$ LUMO $(92.9 \%)$ \\
\hline 4 & 408.30 & 0.0037 & HOMO-3 & $\rightarrow \quad$ LUMO $(98.4 \%)$ \\
\hline 5 & 394.76 & 0.0334 & HOMO-4 & $\rightarrow \quad$ LUMO $(98.5 \%)$ \\
\hline
\end{tabular}


Table S8. Molecular orbital energies (in eV) and molecular orbital distributions (in \%) of $\mathbf{C 3}$ at the ground state $\left(\mathrm{S}_{0}\right)$ and first singlet excited state $\left(\mathrm{S}_{1}\right)$ optimized geometries in THF

\begin{tabular}{ccccc}
\hline & $\mathrm{E}(\mathrm{eV})$ & $o$-carborane & phenyl & carbazole \\
\hline LUMO+3 & -0.44 & $\mathrm{~S}_{0}$ & & 6.8 \\
LUMO+2 & -0.60 & 7.0 & 86.2 & 49.6 \\
LUMO+1 & -1.09 & 19.1 & 6.3 & 88.2 \\
LUMO & -1.35 & 5.0 & 36.5 & 18.8 \\
HOMO & -5.82 & 44.7 & 0.1 & 97.0 \\
HOMO-1 & -6.11 & 2.9 & 0.2 & 98.5 \\
HOMO-2 & -7.10 & 1.3 & 41.2 & 50.9 \\
HOMO-3 & -7.15 & 7.9 & 77.7 & 15.6 \\
\hline LUMO+3 & -0.49 & 6.6 & & \\
LUMO+2 & -0.59 & $\mathrm{~S}_{1}$ & 86.9 & 0.6 \\
LUMO+1 & -0.97 & 12.4 & 80.6 & 12.8 \\
LUMO & -3.26 & 6.6 & 3.1 & 92.6 \\
HOMO & -5.51 & 4.3 & 14.3 & 2.2 \\
HOMO-1 & -6.09 & 83.5 & 0.0 & 93.4 \\
HOMO-2 & -6.88 & 6.5 & 0.0 & 9.1 \\
HOMO-3 & -7.24 & 0.8 & 0.1 & \\
\hline
\end{tabular}




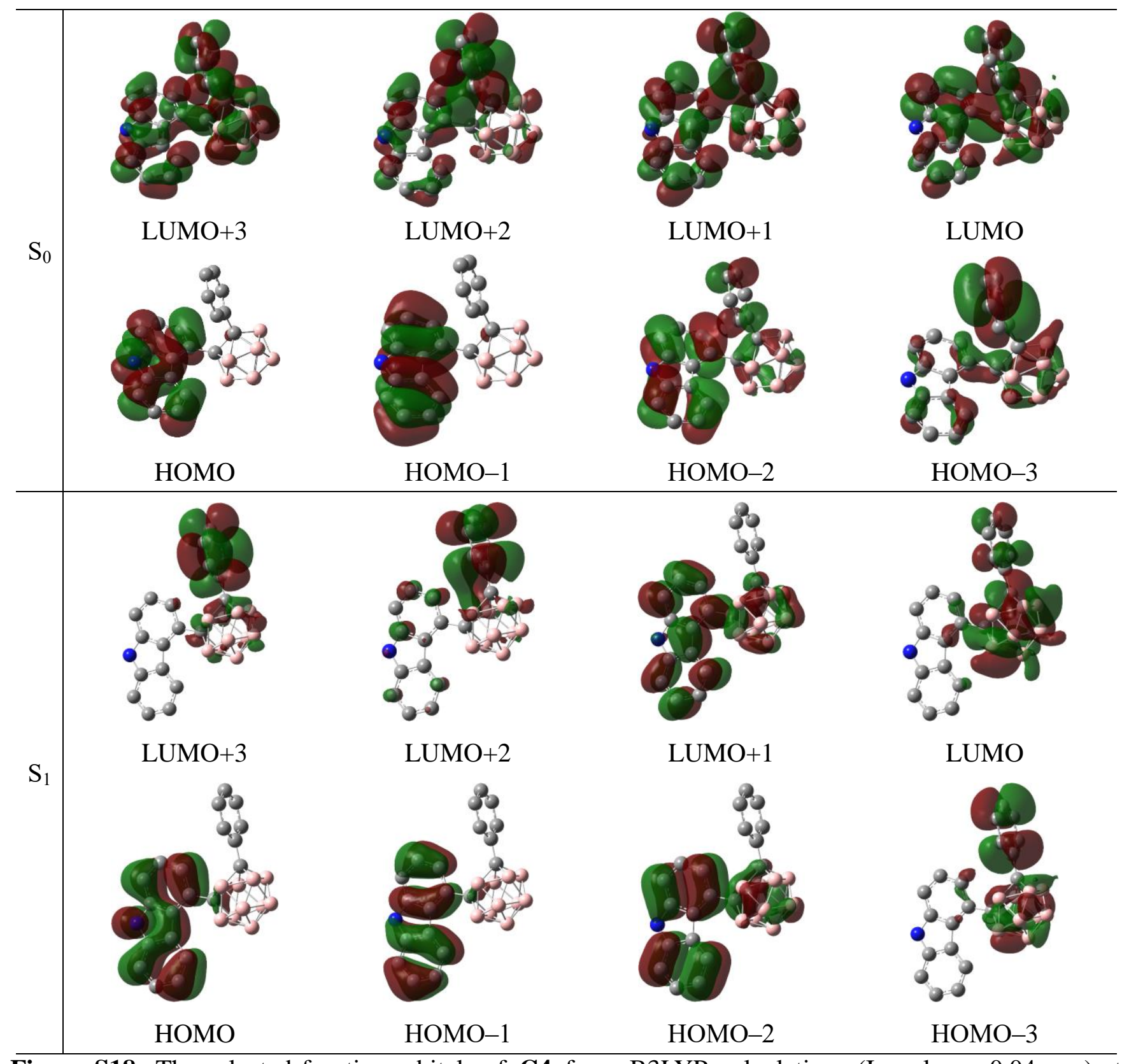

Figure S18. The selected frontier orbitals of C4 from B3LYP calculations (Isovalue $=0.04$ a.u.) at the ground state $\left(S_{0}\right)$ and first singlet excited state $\left(S_{1}\right)$ optimized geometries in THF. 
Table S9. Computed absorption wavelengths $\left(\lambda_{\text {calc }}\right.$ in $\left.\mathrm{nm}\right)$ and oscillator strengths $\left(f_{\text {calc. }}\right)$ for $\mathbf{C 4}$ from TD-B3LYP calculations using the B3LYP geometries at the ground state $\left(\mathrm{S}_{0}\right)$ and first singlet excited state $\left(\mathrm{S}_{1}\right)$ optimized geometries in THF

\begin{tabular}{|c|c|c|c|c|}
\hline state & $\lambda_{\text {calc }}(/ \mathrm{nm})$ & $f_{\text {calc }}$ & \multicolumn{2}{|c|}{ Major contribution } \\
\hline \multicolumn{5}{|c|}{$\mathrm{S}_{0}$} \\
\hline 1 & 347.28 & 0.1669 & HOMO & $\rightarrow$ LUMO $(97.7 \%)$ \\
\hline 2 & 312.40 & 0.0515 & HOMO-1 & $\rightarrow \quad$ LUMO $(95.1 \%)$ \\
\hline 3 & 300.08 & 0.0248 & HOMO & $\rightarrow$ LUMO+1 $(94.8 \%)$ \\
\hline 4 & 274.25 & 0.0233 & HOMO-1 & $\rightarrow$ LUMO+1 $(69.6 \%)$ \\
\hline \multirow{4}{*}{5} & & & HOMO & $\rightarrow$ LUMO+2 (19.9\%) \\
\hline & 263.03 & 0.1207 & HOMO-2 & $\rightarrow$ LUMO $(21.6 \%)$ \\
\hline & & & HOMO-1 & $\rightarrow$ LUMO+1 (19.1\%) \\
\hline & & & HOMO & $\rightarrow$ LUMO+2 $(56.0 \%)$ \\
\hline \multicolumn{5}{|c|}{$\mathrm{S}_{1}$} \\
\hline 1 & 597.16 & 0.1981 & HOMO & $\rightarrow \quad$ LUMO $(98.7 \%)$ \\
\hline 2 & 525.74 & 0.0035 & HOMO-1 & $\rightarrow$ LUMO $(98.7 \%)$ \\
\hline 3 & 510.66 & 0.0007 & HOMO-2 & $\rightarrow$ LUMO $(94.6 \%)$ \\
\hline 4 & 415.64 & 0.0032 & HOMO-3 & $\rightarrow$ LUMO $(98.8 \%)$ \\
\hline 5 & 402.64 & 0.0916 & HOMO-4 & $\rightarrow \quad$ LUMO $(98.5 \%)$ \\
\hline
\end{tabular}


Table S10. Molecular orbital energies (in eV) and molecular orbital distributions (in \%) of $\mathbf{C 4}$ at the ground state $\left(\mathrm{S}_{0}\right)$ and first singlet excited state $\left(\mathrm{S}_{1}\right)$ optimized geometries in THF

\begin{tabular}{ccccc}
\hline & $\mathrm{E}(\mathrm{eV})$ & $o$-carborane & phenyl & carbazole \\
\hline LUMO+3 & -0.29 & $\mathrm{~S}_{0}$ & 27.7 & 38.5 \\
LUMO+2 & -0.56 & 11.6 & 65.0 & 23.4 \\
LUMO+1 & -1.04 & 16.1 & 40.2 & 43.7 \\
LUMO & -1.72 & 40.2 & 14.5 & 45.3 \\
HOMO & -5.75 & 1.5 & 0.1 & 98.3 \\
HOMO-1 & -6.13 & 1.3 & 0.3 & 98.4 \\
HOMO-2 & -7.09 & 6.7 & 17.3 & 76.0 \\
HOMO-3 & -7.14 & 10.2 & 80.0 & 9.8 \\
\hline LUMO+3 & -0.48 & $\mathrm{~S}_{1}$ & & \\
LUMO+2 & -0.60 & 11.5 & 87.8 & 0.8 \\
LUMO+1 & -1.13 & 8.1 & 84.7 & 7.3 \\
LUMO & -3.29 & 15.7 & 0.5 & 83.8 \\
HOMO & -5.52 & 84.2 & 13.2 & 2.6 \\
HOMO-1 & -6.10 & 2.6 & 0.0 & 97.4 \\
HOMO-2 & -6.81 & 1.7 & 0.0 & 98.3 \\
HOMO-3 & -7.22 & 13.0 & 17.6 & 0.2 \\
\hline
\end{tabular}

